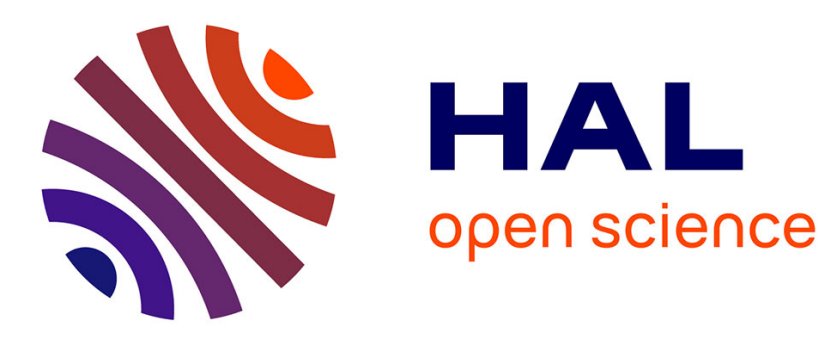

\title{
The Three-Wave Resonant Interaction Equations: Spectral and Numerical Methods
}

Antonio Degasperis, Matteo Conforti, Fabio Baronio, Stefan Wabnitz, Sara Lombardo

\section{- To cite this version:}

Antonio Degasperis, Matteo Conforti, Fabio Baronio, Stefan Wabnitz, Sara Lombardo. The ThreeWave Resonant Interaction Equations: Spectral and Numerical Methods. Letters in Mathematical Physics, 2011, 96 (1-3), pp.367-403. 10.1007/s11005-010-0430-4 . hal-02395151

\section{HAL Id: hal-02395151 https://hal.science/hal-02395151}

Submitted on 5 Dec 2019

HAL is a multi-disciplinary open access archive for the deposit and dissemination of scientific research documents, whether they are published or not. The documents may come from teaching and research institutions in France or abroad, or from public or private research centers.
L'archive ouverte pluridisciplinaire HAL, est destinée au dépôt et à la diffusion de documents scientifiques de niveau recherche, publiés ou non, émanant des établissements d'enseignement et de recherche français ou étrangers, des laboratoires publics ou privés. 


\title{
The three wave resonant interaction equations: spectral and numerical methods
}

\author{
Antonio Degasperis ${ }^{2}$, Matteo Conforti ${ }^{1}$, Fabio Baronio ${ }^{1}$, \\ Stefan Wabnitz ${ }^{1}$, Sara Lombardo ${ }^{3}$ \\ ${ }^{1}$ CNISM and Dipartimento di Ingegneria dell'Informazione, Università di Brescia, \\ Italy \\ E-mail: \\ baronio@ing.unibs.it, matteo.conforti@ing.unibs.it, stefano.wabnitz@ing.unibs.it \\ 2 Dipartimento di Fisica, Università di Roma "La Sapienza", \\ and Istituto Nazionale di Fisica Nucleare, Sezione di Roma, Italy \\ E-mail: antonio.degasperis@roma1.infn.it \\ 3 Department of Mathematics, Vrije Universiteit, Amsterdam, The Netherlands \\ Email: sara@few.vu.nl \\ and School of Mathematics, Alan Turing Building, University of Manchester, UK \\ Email: sara.lombardo@manchester.ac.uk
}

\begin{abstract}
The spectral theory of the integrable partial differential equations which model the resonant interaction of three waves is considered with the purpose of numerically solving the spectral problem for both vanishing and non vanishing boundary values. Methods of computing both the continuum spectrum data and the discrete spectrum eigenvalues are given together with examples of such computations. The explicit spectral representation of the Manley-Rowe invariants is also displayed.
\end{abstract}

Key words: 3 wave resonant interaction, spectral theory, numerical computation MSC: 74J30, 37K15, 65Z05

\section{Introduction}

The discovery and development of soliton theory had, and still has, a deep impact in physics and applied mathematics. This theory has made possible the spectral investigation of several nonlinear partial differential equations (PDEs) with universal applicability. The classic paper by Ablowitz, Kaup, Newell and Segur [1] synthesized a fundamental new idea in the field of theoretical soliton physics. Their approach, which they called the inverse scattering transform (IST), evolved from the pioneering works of Gardner, Greene, 
Kruskal and Miura (GGKM) [2] and of Zakharov and Shabat [3]. The essential idea is that IST is a nonlinear generalization of the ordinary, linear, Fourier transform to the solution of particular nonlinear partial differential (wave) equations with infinite-line boundary conditions. GGKM solved the Kortegde Vries (KdV) equation using a linear integral equation due to Gelfan'd, Levitan, and Marchenko (GLM) [4]; a key step in their approach was to associate a particular (spectral) eigenvalue problem, namely the time-independent Schroedinger equation, with KdV. Zakharov and Shabat applied a similar technique to the nonlinear Schroedinger (NLS) equation [3], for a different eigenvalue problem, and found the exact spectral solution to NLS in terms of two coupled GLM-type equations. AKNS then generalized the method to a large class of wave equations in one space and one time $(1+1)$ dimensions. The theoretical formulation of IST has since been extended to include the modified $\mathrm{KdV}$ [5], the cylindrical KdV [6], Klein-Gordon equations [7-10], the Reduced Maxwell-Bloch equations [11], the self-induced-transparency (SIT) equations $[12,13]$, the coupled NLS equation [14], the massive Thirring model $[15,16]$, the Harry-Dym equation [17], and the three-wave interaction equations [18-20]. For reviews on inverse scattering theory and its application to the solution of integrable PDEs the reader is referred to the literature [21-23]. The focus in the present paper is on the spectral theory of the three-wave interaction equation with the purpose of numerically integrating the spectral equation for both vanishing and non vanishing boundary values. In particular we apply spectral techniques to recently found theoretical and experimental results on the three-wave resonant interaction processes [24-32].

The paper is organized as follows. In section 2 we shortly review the threewave resonant interaction (3WRI) model. In section 3 we discuss the direct spectral problem for both the discrete and the continuous parts of the spectrum when the boundary values of the three wave amplitudes are vanishing. In section 4 we consider the spectral problem in the case in which these boundary values are instead nonvanishing. Both sections 3 and 4 include also the numerical algorithm which has been designed to compute the discrete eigenvalues as well as the scattering coefficients on the continuum spectrum. Section 5 is devoted to numerical examples which illustrate the accuracy of the computational methods by comparing numerical with analytical results. The last section 6 contains concluding remarks.

\section{Three-wave resonant interaction}

It is well-known that three quasi-monochromatic waves with wave-numbers $k_{1}, k_{2}, k_{3}$ and frequencies $\omega_{1}, \omega_{2}, \omega_{3}$ which propagate in a dispersive nonlinear medium with quadratic nonlinearity, interact, and exchange energy, with 
each other if the resonance conditions

$$
k_{1}+k_{2}+k_{3}=0, \omega_{1}+\omega_{2}+\omega_{3}=0 .
$$

are satisfied. Standard multiscale analysis proves that the wave amplitude envelopes $E_{1}(x, t), E_{2}(x, t)$ and $E_{3}(x, t)$ in a one dimensional medium satsfy the following partial differential equations

$$
\left(\frac{\partial}{\partial t}+V_{n} \frac{\partial}{\partial x}\right) E_{n}=\eta_{n} E_{n+1}^{*} E_{n+2}^{*}, n=1,2,3, \bmod 3 .
$$

This system of equations possess two very important properties, namely it is universal and integrable. The first feature, universality, implies that the equations (2) apply, and have been applied, to a variety of different contexts such as fluid dynamics, optics and plasma physics. The second one, integrability, give us mathematical tools to investigate several problems such as the evolution of given initial data, construction of particular analytic solutions (f.i. solitons) and the derivation of (infinitely many) conservation laws.

As for the notation we adopt here and in the following, the parameters $V_{1}$, $V_{2}, V_{3}$, are the characteristic velocities, while the variables $x$ and, respectively, $t$ stand for space coordinate and, respectively, time. However, it should be pointed out that the wave equations (2) are non dispersive since they are first order and therefore an arbitrary linear transformation of the coordinates $(x, t)$ to other coordinates $\left(x^{\prime}, t^{\prime}\right)$ does not change the structure of the equations (2) but only their coefficients, with the implication that the physical meaning of the variables $x$ and $t$ may depend on the particular application one has in mind. In any case we assume that the variable $x$ be chosen in such a way that the profile of two waves, for instance $E_{1}(x, t)$ and $E_{2}(x, t)$, is well localized in the coordinate $x$, namely

$$
E_{1}(x, t) \rightarrow 0, E_{2}(x, t) \rightarrow 0, x \rightarrow \pm \infty,
$$

while the profile of the third wave $E_{3}(x, t)$ may be either as well localized,

$$
E_{3}(x, t) \rightarrow 0, x \rightarrow \pm \infty,
$$

or superimposed to a non vanishing background, say

$$
\left|E_{3}(x, t)\right| \rightarrow \text { constant } \neq 0, x \rightarrow \pm \infty .
$$

This assumption plays a relevant role in the following, as our numerical methods deal with the spectral theory in the variable $x$ while the variable $t$ remains the evolution variable. The case with the boundary conditions (3) and (4) describes the propagation of three bright pulses which, if far apart from each other, propagate at their characteristic speed without dispersion, and it will be referred to as the BBB case. In the other case the boundary conditions 
(3) and (5) describe two bright (in this instance $E_{1}$ and $E_{2}$ ) and one dark $\left(E_{3}\right)$ pulses and we refer to it as the BBD case. Note that in this case the two bright pulses, because of their persistent interaction with the background, propagate instead with dispersion. Finally the constant parameters $\eta_{1}, \eta_{2}, \eta_{3}$ in front of the quadratic terms, namely the coupling constants, are just signs, i.e. $\eta_{n}^{2}=1$. These coefficients are so taken because only the signs of them are relevant. Indeed the modulus of the coupling constants can be arbitrarily changed by a scaling transformation of the amplitudes, namely $E_{n} \rightarrow \alpha_{n} E_{n}$ where $\alpha_{1}, \alpha_{2}, \alpha_{3}$ are arbitrary complex constants. We also point out that here and in the following the labeling of the three waves is conventionally fixed by the ordering

$$
V_{1}<V_{2}<V_{3}
$$

of the characteristic velocities. The three signs $\eta_{1}, \eta_{2}, \eta_{3}$ can be freely chosen, and this implies that, once the velocities $V_{n}$ are fixed, one remains with $2^{3}$ cases. These however reduce to four because the transformation $E_{n} \rightarrow-E_{n}$ induces the change $\eta_{n} \rightarrow-\eta_{n}$. However we prefer to include all these cases in our discussion by using the notation

$$
\eta_{1} \eta_{2} \eta_{3}=\eta, \eta= \pm 1
$$

which separates the four cases with $\eta=1$ from the other fully equivalent four cases with $\eta=-1$. Different physical processes modelled by the 3WRI equations correspond to different choices of the three signs $\eta_{1}, \eta_{2}, \eta_{3}$. Three non equivalent types of processes have been identified as explosive, stimulated backscatter and soliton exchange type. If we consider for instance the cases with $\eta=1$ (see (7)) the explosive type corresponds to $\left(\eta_{1}, \eta_{2}, \eta_{3}\right)=$ $(+,+,+)$. The backscatter type corresponds to the two cases $\left(\eta_{1}, \eta_{2}, \eta_{3}\right)=$ $(+,-,-)$ and $(-,-,+)$ and are equivalent to each other as a transformation of (2) exists which changes both the dependent and independent variables and takes one of these two cases into the other. The case of soliton exchange type occurs if $\left(\eta_{1}, \eta_{2}, \eta_{3}\right)=(-,+,-)$. In the following the signs $\eta_{n}$ will be left free so as to deal with all different types of interaction. However it should be kept in mind that the explosive, backscatter and soliton exchange interactions describe quite different physical processes [37].

We briefly recall the general features of these three cases. Consider first the $\mathrm{BBB}$ case and assume that time is so large in the past that the three pulses do not overlap. Assume also that the wave $E_{2}$ contains solitons, then a general feature of $3 \mathrm{WRI}$ is the instability of the solitons contained in the middle velocity wave $E_{2}$ : during the interaction the wave $E_{2}$ releases all its solitons to both the fast and the slow waves. In this process the number of solitons is conserved, provided we double count the solitons in the envelope wave $E_{2}$. In the explosive case, only the wave $E_{2}$ can contain solitons, whereas slow and fast waves, $E_{1}$ and, respectively, $E_{3}$ can not. In this case if wave $E_{2}$ has enough energy to sustain at least one soliton, a singularity appears in a finite time 
since no soliton is allowed to be in the spectrum of the fast and slow waves. In the soliton exchange case all three waves may contain solitons; if wave $E_{2}$ has enough energy to sustain at least one soliton, the soliton in wave $E_{2}$ decays into solitons in the waves $E_{1}$ and $E_{3}$. The reverse process, i.e. the up-conversion of solitons from waves $E_{1}$ and $E_{3}$ into wave $E_{2}$, is also possible; however this process is unstable, namely the lifetime of wave $E_{2}$ is finite. In the backscatter case a bright soliton can propagate in a stable fashion in the slow wave (if the signs are $(-,-,+))$ or in the fast wave (if the signs are $(+,-,-)$ ), namely no soliton exchange can take place. For a detailed description of these processes the reader is referred to [37].

In the case of $\mathrm{BBD}$ solutions the behavior of the three waves is quite different with respect to the $\mathrm{BBB}$ case. This is due to the nonvanishing of the wave $E_{3}(x, t)$ as $|x| \rightarrow \infty$, see (5). In fact, even if far apart from each other, two bright wave packets $E_{1}(x, t)$ and $E_{2}(x, t)$ interact with the background $E_{3}(x, t)$ at all times. If we assume that the signs are $\eta_{1}=\eta_{3}=-\eta_{2}=1$ in (2), this interaction generically causes these two bright pulses to asymptotically disperse away. In this respect, it is worth noticing that the time evolution modeled by the 3WRI equations (2) with the present choice of signs (which is equivalent to the focusing Nonlinear Schroedinger equation) makes the plane wave background $E_{3}(x, t)$ stable, an effect which is opposite of that of the Nonlinear Schroedinger equation which makes instead the plane wave unstable. Only for soliton solutions this dispersion combines with the nonlinearity to form a triplet of locked non dispersive pulses (also termed simulton) which travels with the same velocity. This soliton velocity $V$ is different from the three characteristic velocities $V_{1}, V_{2}, V_{3}$. If the simulton velocity $V$ is sufficiently high, larger than a critical value, the simulton is unstable and decays by emitting a stable bump (a soliton itself) of the background cw $E_{3}(x, t)$ which travels with velocity $V_{3}$. Because of this emission process, the simulton velocity decreases below the critical value and the resulting simulton becomes stable. The entire emission process, and its reversed one, namely the absorption of the $E_{3}$ stable soliton, is fully and explicitly described by the so-called boomeron solution whose analytic expression is known $[27,28]$. Details and theoretical and experimental results may be found in $[27,28]$.

\section{BBB waves}

The integrability of the $3 \mathrm{WRI}$ equations (2) follows from the fact that these equations are the compatibility conditions of two $3 \times 3$ matrix Ordinary Differential Equations (ODEs), one in the variable $x$ and the other one in $t$. Indeed this fact has important consequences. Among others, and as relevant to our present purposes, it gives a way to set up a nonlinear generalization of the Fourier analysis of solutions of the associated initial value prob- 
lem. In particular, this generalization leads to decompose a given solution $E_{1}(x, t), E_{2}(x, t), E_{3}(x, t)$ as functions of $x$ at any fixed time $t$ in its continuum spectrum component (radiation) and discrete spectrum component (solitons). We find it convenient to start our analysis by considering first the more general system of six, rather than three, PDEs

$$
\begin{gathered}
\left(\frac{\partial}{\partial t}+V_{n} \frac{\partial}{\partial x}\right) u_{n}=\left(V_{n+1}-V_{n+2}\right) v_{n+1} v_{n+2}, n=1,2,3, \bmod 3 \\
\left(\frac{\partial}{\partial t}+V_{n} \frac{\partial}{\partial x}\right) v_{n}=-\left(V_{n+1}-V_{n+2}\right) u_{n+1} u_{n+2}, n=1,2,3, \bmod 3
\end{gathered}
$$

for the complex variables $u_{n}$ and $v_{n}, n=1,2,3$, and then to investigate (see (3.4)) the 3WRI equations (2) as a reduction of this larger system by expressing the solutions $u_{n}(x, t)$ and $v_{n}(x, t)$ of the unreduced system $(8)$ in terms of the solutions $E_{n}(x, t)$ of the $3 \mathrm{WRI}$ equations (2). Indeed the unreduced system (8) is also integrable as it is the condition that the pair of the $3 \times 3$ matrix ODEs (a subscripted variable denotes partial differentiation)

$$
\begin{gathered}
\psi_{x}=[i \lambda A+E(x, t)] \psi, \\
\psi_{t}=[i \lambda B+F(x, t)] \psi+\psi C,
\end{gathered}
$$

be compatible with each other. Here $C$ is a $x$-independent matrix which depends on the particular boundary values given on the $x$-axis (see below), $\psi=\psi(x, t, \lambda)$ is a common 3 -dim vector solution and $\lambda$ is the complex spectral variable. $A$ and $B$ are constant, real and traceless diagonal matrices, $A=\operatorname{diag}\left\{a_{1}, a_{2}, a_{3}\right\}, B=\operatorname{diag}\left\{b_{1}, b_{2}, b_{3}\right\}$,

$$
a_{n}=a_{n}^{*}, \quad b_{n}=b_{n}^{*}, a_{1}+a_{2}+a_{3}=0, b_{1}+b_{2}+b_{3}=0,
$$

whose entries define the group velocities $V_{n}$, namely

$$
V_{n}=-\frac{b_{n+1}-b_{n+2}}{a_{n+1}-a_{n+2}}, n=1,2,3, \bmod 3 .
$$

They can be conveniently chosen as

$$
\begin{gathered}
a_{n}=2 V_{n}-V_{n+1}-V_{n+2}, n=1,2,3, \bmod 3, \\
b_{n}=2 V_{n+1} V_{n+2}-V_{n}\left(V_{n+1}+V_{n+2}\right), n=1,2,3, \bmod 3 .
\end{gathered}
$$

and we note, for future reference, that the ordering (6) of the group velocities implies that

$$
a_{1}<a_{2}<a_{3}, a_{1}<0, a_{3}>0 .
$$

The six wave fields $u_{n}$ and $v_{n}$ are the entries of the matrix $E(x, t)$,

$$
E=\left(\begin{array}{ccc}
0 & u_{3} & v_{2} \\
v_{3} & 0 & u_{1} \\
u_{2} & v_{1} & 0
\end{array}\right)
$$


and the matrix $F(x, t)$ takes the expression

$$
F=-\left(\begin{array}{lrr}
0 & V_{3} u_{3} & V_{2} v_{2} \\
V_{3} v_{3} & 0 & V_{1} u_{1} \\
V_{2} u_{2} & V_{1} v_{1} & 0
\end{array}\right)
$$

Since we now deal with the spectral method of solution, hereafter we omit to explicitly show the dependence on the time variable $t$ (whose value is taken fixed), and we focus our attention only on the complex variable $\lambda$ and on the variable $x$, with respect to which the solution $\psi(x, \lambda)$ satisfies the differential equation (9), namely

$$
\psi_{x}=[i \lambda A+E(x)] \psi,-\infty<x<+\infty,
$$

with the condition that the matrix $E(x)$ is everywhere bounded and vanishes sufficiently fast as $x \rightarrow \pm \infty$ (see the conditions (3) and (4) for BBB waves). As for the spectral variable $\lambda$, one defines instead a matrix Riemann-Hilbert problem in the complex $\lambda$-plane with appropriate spectral data which are the nonlinear analog of the Fourier transform of the matrix $E(x)$. The computation of these spectral data corresponding to the matrix $E(x)$ is the direct problem, and it amounts to integrating the differential equation (18) (see below). The recovering of the matrix $E(x)$ from the spectral data is the inverse problem, and it amounts to solve the Riemann-Hilbert problem. The way to solve these two problems is the main tool to investigate the initial value problem associated with the 3WRI equations (2). The treatment of the direct and inverse problems is given in [20] (see also) and it is not fully reported here. Below we merely limit our attention to those spectral data of the matrix $E(x)$ whose numerical computation may prove to be useful to understanding wave interaction processes. For instance, the numerical computation of the discrete eigenvalues gives the soliton content of the wave fields. This aspect is specially evident in the spectral decomposition of the constants of the motion of the 3 WRI equations (2) we derive in this section (see subsection (3.3)).

\subsection{Continuum spectrum}

We first set up our notation and provide the basic facts whose proof is either straight or follows by standard techniques (f.i. see [20]).

It is essential to introduce first the differential equation

$$
\tilde{\psi}_{x}=\left[-i \lambda A-E^{T}(x)\right] \tilde{\psi},-\infty<x<+\infty,
$$

which is defined as the adjoint equation of (18). We note that the adjoint equation (19) follows from (18) by the rule $A \rightarrow-A, E \rightarrow-E^{T}$ (here- 
after $M^{T}$ is the transposed of the matrix $M$ ), and that therefore the adjoint equation of the adjoint equation is the original equation itself. This definition is motivated by the following property: if $\psi$ and $\tilde{\psi}$ are arbitrary vector, or matrix, solutions of respectively, (18) and (19), then $\tilde{\psi}^{T}(x, \lambda) \psi(x, \lambda)$, and of course also $\psi^{T}(x, \lambda) \tilde{\psi}(x, \lambda)$, is $x$-independent, namely

$$
\frac{\partial}{\partial x}\left[\tilde{\psi}^{T}(x, \lambda) \psi(x, \lambda)\right]=0
$$

In the following discussion it is expedient to treat the differential equation (18) and its adjoint equation (19) in a parallel way. The following remark is also relevant to our treatment of the spectral problem. The spectrum, both continuum and discrete, as well as the spectral data defined on it should be invariant with respect to the transformation

$$
a_{n} \rightarrow a_{n}+a \quad, \quad n=1,2,3,
$$

for any real value of the parameter $a$. In fact, the condition $\operatorname{tr} A=0$, see (11), which we maintain through this paper, is just convenient but not essential. Let us consider first the continuum spectrum, namely the real values of the spectral variable $\lambda$. If $\lambda$ is real, then any solution of the differential equations (18) and (19) is bounded and, if $|x|$ is sufficiently large, is oscillating in the variable $x$. This yields the following definition: for any real value of $\lambda$, the " left " $\Psi_{L}(x, \lambda)$ and "right " $\Psi_{R}(x, \lambda)$ matrix solutions of $(18)$, and the "left" $\tilde{\Psi}_{L}(x, \lambda)$ and " right " $\tilde{\Psi}_{R}(x, \lambda)$ matrix solutions of the adjoint equation (19) are uniquely defined by the asymptotic conditions

$$
\begin{array}{cc}
\Psi_{L}(x, \lambda) \longrightarrow \exp (i \lambda x A) \quad, \quad x \rightarrow-\infty \\
\Psi_{R}(x, \lambda) \longrightarrow \exp (i \lambda x A) \quad, \quad x \rightarrow+\infty \\
\tilde{\Psi}_{L}(x, \lambda) \longrightarrow \exp (-i \lambda x A), & x \rightarrow-\infty \\
\tilde{\Psi}_{R}(x, \lambda) \longrightarrow \exp (-i \lambda x A), & x \rightarrow+\infty
\end{array}
$$

Since the determinant of any matrix solution of (18) and of (19) is $x$-independent if $\operatorname{tr} A=0$, the conditions (22) and (23) imply that

$$
\operatorname{det} \Psi_{L}(x, \lambda)=\operatorname{det} \Psi_{R}(x, \lambda)=\operatorname{det} \tilde{\Psi}_{L}(x, \lambda)=\operatorname{det} \tilde{\Psi}_{R}(x, \lambda)=1 .
$$

Therefore $\Psi_{L}$ and $\Psi_{R}$ are two fundamental solutions of (18), and the matrix $\Psi_{R}^{-1} \Psi_{L}$ has to be $x$-independent, with the implication that

$$
\Psi_{L}(x, \lambda)=\Psi_{R}(x, \lambda) S(\lambda) .
$$

Similarly, for the adjoint equation (19) we have

$$
\tilde{\Psi}_{L}(x, \lambda)=\tilde{\Psi}_{R}(x, \lambda) \tilde{S}(\lambda)
$$


The $3 \times 3$ matrices $S(\lambda)$ and $\tilde{S}(\lambda)$ are well defined on the real axis of the complex $\lambda$-plane (i.e. the continuum spectrum of the differential equations (18) and (19)), and are known as scattering matrices associated to the matrix $E(x)$. From the equations (24) there immediately follow the important properties

$$
\operatorname{det} S(\lambda)=\operatorname{det} \tilde{S}(\lambda)=1
$$

Since the two differential equations (18) and (19) are each the adjoint equation of the other, their solutions are related to each other in the way displayed by (20), which in particular implies the important relations

$$
\tilde{\Psi}_{L}(x, \lambda)=\Psi_{L}^{T-1}(x, \lambda), \quad \tilde{\Psi}_{R}(x, \lambda)=\Psi_{R}^{T-1}(x, \lambda) .
$$

Moreover combining these formulae with (25) and (26) leads to the following relation

$$
S^{T}(\lambda) \tilde{S}(\lambda)=\mathbb{I}
$$

among the two scattering matrices.

\subsection{Discrete spectrum}

Let us consider now the discrete spectrum. We first observe that, since the discrete spectrum is required to be invariant with respect to the change (21) where $a$ is a real arbitrary number, the definition of, and search for, a discrete eigenvalue requires some care. Thus the definition is: a complex number $\lambda$ is a discrete eigenvalue of the spectral equation (18) if a real number $a$ exists such that the ODE

$$
\psi_{x}=[i \lambda(A+a \mathbf{1})+E(x)] \psi,
$$

possesses a vector solution in $L_{2}(-\infty,+\infty)$. The discrete eigenvalues are then associated with the existence of an everywhere bounded vector solution of the ODE (30) which exponentially decreases at both $x=-\infty$ and $x=+\infty$. Therefore, because of this exponential decay of the solution at large $|x|$, a discrete eigenvalue $\lambda$ has to be complex, i.e. with $\operatorname{Im} \lambda \neq 0$. It is plain that the discrete eigenvalues of the adjoint spectral equation (19) are similarly defined. The computation of the discrete spectrum requires therefore fixing the value of $a$ and then looking, for each fixed value of $a$, for $L_{2}$ solutions of (30). In practice, by taking into account the inequalities (15), only two values of $a$ have to be considered. Indeed, if we consider the spectral equation (30), the generic solution $\psi$ has the asymptotic exponential behavior

$$
\psi(x, \lambda) \rightarrow\left(\begin{array}{c}
c_{ \pm}^{(1)}(\lambda) e^{i \lambda x\left(a_{1}+a\right)} \\
c_{ \pm}^{(2)}(\lambda) e^{i \lambda x\left(a_{2}+a\right)} \\
c_{ \pm}^{(3)}(\lambda) e^{i \lambda x\left(a_{3}+a\right)}
\end{array}\right), x \rightarrow \pm \infty
$$


where three of the six complex coefficients $c_{ \pm}^{(n)}$ can be arbitrarily given (f.i. at $x=-\infty$ or at $x=+\infty)$. As a consequence, there are no eigenvalues if $a<-a_{3}$ or if $a>-a_{1}$. Therefore the parameter $a$ can take its value only in the interval $-a_{3}<a<-a_{1}$. Moreover, since $a_{1}+a<0$ and $a_{3}+a>0$, one is left with only two cases of interest, namely $a_{2}+a<0$ and $a_{2}+a>0$. In conclusion, eigenvalues should be looked for only when $a$ is either in the subinterval $-a_{3}<a<-a_{2}$ or in the subinterval $-a_{2}<a<-a_{1}$. The specific value of $a$ in each one of these two intervals does not matter. However, since the value of $a$ characterizes the rapidity of the exponential decay (or growth) of the solution $\psi$, see (31), choosing the "optimal" values $a=-\frac{1}{2}\left(a_{2}+a_{3}\right)$ in the first interval,and $a=-\frac{1}{2}\left(a_{1}+a_{2}\right)$ in the second interval, may be convenient to the purpose of numerically integrating the equation (30). If we assume f.i. that the eigenvalue $\lambda$ is in the upper half-plane of the complex plane, $\operatorname{Im} \lambda>0$, then the actual computation of eigenvalues goes via the following steps:

(1) choose $a=-\frac{1}{2}\left(a_{2}+a_{3}\right)$ and integrate (30) by assuming the initial conditions $c_{1}^{(+)}=c_{2}^{(+)}=0$ and $c_{3}^{(+)}=1$,

(2) compute $c_{3}^{(-)}(\lambda)$,

(3) find the zeros $\lambda^{(+)}$(if any) of $c_{3}^{(-)}(\lambda)$, say $c_{3}^{(-)}\left(\lambda^{(+)}\right)=0$,

(4) choose $a=-\frac{1}{2}\left(a_{1}+a_{2}\right)$ and integrate (30) by assuming the initial conditions $c_{2}^{(-)}=c_{3}^{(-)}=0$ and $c_{1}^{(-)}=1$,

(5) compute $c_{1}^{(+)}(\lambda)$,

(6) find the zeros $\lambda^{(+)}$(if any) of $c_{1}^{(+)}(\lambda)$, say $c_{1}^{(+)}\left(\lambda^{(+)}\right)=0$.

The eigenvalues computed by the steps (1), (2) and (3) may be termed righteigenvalues because they require integrating the spectral equation (30) from the right (i.e. from $x=+\infty$ ), while in the other case those eigenvalues computed via steps (4), (5) and (6) may be referred to as left-eigenvalues. A similar scheme of computation, with obvious changes, has to be carried out in the lower half-plane, namely with $\operatorname{Im} \lambda<0$, in order to completely compute the spectrum $\left\{\lambda^{(+)}, \lambda^{(-)}\right\}$with $\pm \operatorname{Im}\left[\lambda^{( \pm)}\right]>0$. According to our remark above the spectrum consists of two parts, namely the right-spectrum and the left-spectrum. It is also clear that this same procedure applies to the computation of the discrete spectrum, $\left\{\lambda^{(+)}, \lambda^{(-)}\right\}$with $\pm \operatorname{Im}\left[\lambda^{( \pm)}\right]>0$, of the adjoint equation (see (19))

$$
\tilde{\psi}_{x}=-\left[i \lambda(A+a \mathbf{1})+E^{T}(x)\right] \tilde{\psi} .
$$

Next we give the way the discrete spectrum is related to the scattering matrices $S(\lambda)$ and $\tilde{S}(\lambda)$. Since these two matrices are defined on the real axis, first we deal with the analytic continuation off the real axis. To this aim it is standard to transform the ODEs (18) and (19) into Volterra type integral equations, which, for the fundamental matrix solutions $\Psi_{L}(x, \lambda)$ and $\Psi_{R}(x, \lambda)$ of (18), and $\tilde{\Psi}_{L}(x, \lambda)$ and $\tilde{\Psi}_{R}(x, \lambda)$ of the adjoint equation (19) take the following 
expression (see also (22) and (23))

$$
\begin{gathered}
\Phi_{L}(x, \lambda)=\mathbf{1}+\int_{-\infty}^{x} d y e^{i \lambda A(x-y)} E(y) \Phi_{L}(y, \lambda) e^{-i \lambda A(x-y)}=\left(\begin{array}{ccc}
\vdots & \vdots & \vdots \\
\phi_{L}^{(1)} & \phi_{L}^{(2)} & \phi_{L}^{(3)} \\
\vdots & \vdots & \vdots
\end{array}\right)^{(33)} \\
\Phi_{R}(x, \lambda)=\mathbf{1}-\int_{x}^{+\infty} d y e^{i \lambda A(x-y)} E(y) \Phi_{R}(y, \lambda) e^{-i \lambda A(x-y)}=\left(\begin{array}{ccc}
\vdots & \vdots & \vdots \\
\phi_{R}^{(1)} & \phi_{R}^{(2)} & \phi_{R}^{(3)} \\
\vdots & \vdots & \vdots
\end{array}\right)^{(34)} \\
\tilde{\Phi}_{L}(x, \lambda)=\mathbf{1}-\int_{-\infty}^{x} d y e^{-i \lambda A(x-y)} E^{T}(y) \tilde{\Phi}_{L}(y, \lambda) e^{i \lambda A(x-y)}=\left(\begin{array}{ccc}
\tilde{\phi}_{L}^{(1)} & \tilde{\phi}_{L}^{(2)} & \tilde{\phi}_{L}^{(3)} \\
\vdots & \vdots & \vdots \\
& & \\
& \vdots & \\
\vdots & & \vdots \\
\tilde{\phi}_{R}^{(1)} & \tilde{\phi}_{R}^{(2)} & \tilde{\phi}_{R}^{(3)} \\
\vdots & \vdots & \vdots
\end{array}\right),
\end{gathered}
$$

where, for any matrix solution $\Psi$ of (18) and $\tilde{\Psi}$ of (19), we define $\Phi=\Psi e^{-i \lambda A x}$ and $\tilde{\Phi}=\tilde{\Psi} e^{i \lambda A x}$. Moreover, in the four equations above, the vector $\phi^{(n)}$ is the $n$-th column of the matrix $\Phi$. Though these integral equations are well defined for real $\lambda$, by standard arguments (see f.i. [20]) one can prove that these integral equations imply that

- $\phi_{L}^{(1)}(x, \lambda)$ and $\phi_{R}^{(3)}(x, \lambda)$ are analytic for $\operatorname{Im} \lambda>0$,

- $\phi_{R}^{(1)}(x, \lambda)$ and $\phi_{L}^{(3)}(x, \lambda)$ are analytic for $\operatorname{Im} \lambda<0$,

- $\tilde{\phi}_{R}^{(1)}(x, \lambda)$ and $\tilde{\phi}_{L}^{(3)}(x, \lambda)$ are analytic for $\operatorname{Im} \lambda>0$,

- $\tilde{\phi}_{L}^{(1)}(x, \lambda)$ and $\tilde{\phi}_{R}^{(3)}(x, \lambda)$ are analytic for $\operatorname{Im} \lambda<0$.

In general the matrix elements of the scattering matrices $S(\lambda)$ and $\tilde{S}(\lambda)$ are not analytic with the exception of the diagonal entries $S_{11}, S_{33}$ and $\tilde{S}_{11}, \tilde{S}_{33}$ which can instead be analytically continued off the real axis as a consequence of the analyticity properties listed above. Indeed, combining (25), as well as (26), with (28) yields the expressions

$$
S(\lambda)=\tilde{\Psi}_{R}^{T} \Psi_{L}=e^{-i \lambda A x} \tilde{\Phi}_{R}^{T} \Phi_{L} e^{i \lambda A x}, \tilde{S}(\lambda)=\Psi_{R}^{T} \tilde{\Psi}_{L}=e^{i \lambda A x} \Phi_{R}^{T} \tilde{\Phi}_{L} e^{-i \lambda A x}
$$

of the scattering matrices, and, in particular, of their entries

$$
S_{11}(\lambda)=\tilde{\phi}_{R}^{(1) T}(x, \lambda) \phi_{L}^{(1)}(x, \lambda), \quad S_{33}(\lambda)=\tilde{\phi}_{R}^{(3) T}(x, \lambda) \phi_{L}^{(3)}(x, \lambda),
$$




$$
\tilde{S}_{11}(\lambda)=\tilde{\phi}_{L}^{(1) T}(x, \lambda) \phi_{R}^{(1)}(x, \lambda), \quad \tilde{S}_{33}(\lambda)=\tilde{\phi}_{L}^{(3) T}(x, \lambda) \phi_{R}^{(3)}(x, \lambda),
$$

which therefore imply that

- $S_{11}(\lambda)$ and $\tilde{S}_{33}(\lambda)$ are analytic for $\operatorname{Im} \lambda>0$,

- $S_{33}(\lambda)$ and $\tilde{S}_{11}(\lambda)$ are analytic for $\operatorname{Im} \lambda<0$,

a result which is crucial to our aim of relating these scattering matrix entries to the discrete eigenvalues. In fact, applying the method of computing eigenvalues we have step-by-step detailed above (see our discussion following (31)), and taking into account that $S^{-1}(\lambda)=\tilde{S}^{T}(\lambda)$ (see (29)), readily yields the characterization of the discrete eigenvalues and the expression of their corresponding eigenfunctions. This goes a follows: if the eigenvalue $\lambda$ is in the upper half-plane, $\operatorname{Im} \lambda>0$, and $\chi(x)$ is its corresponding eigenfunction of the spectral problem (30), then, if we chose in (31) $a=-\frac{1}{2}\left(a_{2}+a_{3}\right)$,

$$
\tilde{S}_{33}\left(\tilde{\lambda}^{(3)}\right)=0 \quad, \quad \tilde{\chi}^{(3)}(x)=e^{-\frac{i}{2} \tilde{\lambda}^{(3)} x\left(a_{2}+a_{3}\right)} \psi_{R}^{(3)}\left(x, \tilde{\lambda}^{(3)}\right)
$$

while if we choose instead $a=-\frac{1}{2}\left(a_{2}+a_{1}\right)$, we have

$$
S_{11}\left(\lambda^{(1)}\right)=0, \quad \chi^{(1)}(x)=e^{-\frac{i}{2} \lambda^{(1)} x\left(a_{2}+a_{1}\right)} \psi_{L}^{(1)}\left(x, \lambda^{(1)}\right) .
$$

Therefore $\lambda^{(1)}$ and, respectively, $\tilde{\lambda}^{(3)}$ are the eigenvalues in the upper halfplane, and they are the zeros of $S_{11}(\lambda)$ and, respectively, of $\tilde{S}_{33}(\lambda)$. In the lower half plane, $\operatorname{Im} \lambda<0$, we similarly find that, if we chose in (31) $a=-\frac{1}{2}\left(a_{2}+a_{3}\right)$,

$$
S_{33}\left(\lambda^{(3)}\right)=0, \quad \chi^{(3)}(x)=e^{-\frac{i}{2} \lambda^{(3)} x\left(a_{2}+a_{3}\right)} \psi_{L}^{(3)}\left(x, \lambda^{(3)}\right),
$$

while if we choose instead $a=-\frac{1}{2}\left(a_{2}+a_{1}\right)$, we have

$$
\tilde{S}_{11}\left(\tilde{\lambda}^{(1)}\right)=0 \quad, \quad \tilde{\chi}^{(1)}(x)=e^{-\frac{i}{2} \tilde{\lambda}^{(1)} x\left(a_{2}+a_{1}\right)} \psi_{R}^{(1)}\left(x, \tilde{\lambda}^{(1)}\right)
$$

We conclude therefore that the discrete eigenvalues are in one-to-one correspondence with all zeros of the scattering matrix entries $S_{11}(\lambda), \tilde{S}_{33}(\lambda)$ in the upper half-plane $(\operatorname{Im} \lambda>0)$, and with all zeros of the scattering matrix entries $S_{33}(\lambda), \tilde{S}_{11}(\lambda)$ in the lower half-plane $(\operatorname{Im} \lambda<0)$. Moreover we note that $\lambda^{(1)}$ and $\lambda^{(3)}$ are left-eigenvalues while $\tilde{\lambda}^{(1)}$ and $\tilde{\lambda}^{(3)}$ are instead right-eigenvalues. Consider now the adjoint problem, namely the equation (32). Again the same analysis leads to conclude the following: if the eigenvalue $\lambda$ is in the upper half-plane, $\operatorname{Im} \lambda>0$, and $\tilde{\chi}(x)$ is its corresponding eigenfunction, then, if we chose in (32) $a=-\frac{1}{2}\left(a_{2}+a_{3}\right)$,

$$
\tilde{S}_{33}\left(\tilde{\lambda}^{(3)}\right)=0, \tilde{\phi}^{(3)}(x)=e^{\frac{i}{2} \tilde{\lambda}^{(3)} x\left(a_{2}+a_{3}\right)} \tilde{\psi}_{L}^{(3)}\left(x, \tilde{\lambda}^{(3)}\right),
$$

while if we choose instead $a=-\frac{1}{2}\left(a_{2}+a_{1}\right)$, we have

$$
S_{11}\left(\lambda^{(1)}\right)=0 \quad, \quad \phi^{(1)}(x)=e^{\frac{i}{2} \lambda^{(1)} x\left(a_{2}+a_{1}\right)} \tilde{\psi}_{R}^{(1)}\left(x, \lambda^{(1)}\right) .
$$


In the lower half plane, $\operatorname{Im} \lambda<0$, we similarly find that, if we chose in (32) $a=-\frac{1}{2}\left(a_{2}+a_{3}\right)$,

$$
S_{33}\left(\lambda^{(3)}\right)=0, \quad \phi^{(3)}(x)=e^{\frac{i}{2} \lambda^{(3)} x\left(a_{2}+a_{3}\right)} \tilde{\psi}_{R}^{(3)}\left(x, \lambda^{(3)}\right)
$$

while if we choose instead $a=-\frac{1}{2}\left(a_{2}+a_{1}\right)$, we have

$$
\tilde{S}_{11}\left(\tilde{\lambda}^{(1)}\right)=0, \tilde{\phi}^{(1)}(x)=e^{\frac{i}{2} \tilde{\lambda}^{(1)} x\left(a_{2}+a_{1}\right)} \tilde{\psi}_{L}^{(1)}\left(x, \tilde{\lambda}^{(1)}\right) .
$$

We conclude therefore that the discrete spectrum of the adjoint equation (32) coincides with the previously found spectrum of the equation (30), while their corresponding eigenfunctions, $\phi^{(1)}(x), \phi^{(3)}(x), \tilde{\phi}^{(1)}(x), \tilde{\phi}^{(3)}(x)$, take an expression which is different from that of the eigenfunctions $\chi^{(1)}(x), \chi^{(3)}(x), \tilde{\chi}^{(1)}(x), \tilde{\chi}^{(3)}(x)$ of the equation (30). In this case $\lambda^{(1)}$ and $\lambda^{(3)}$ are right-eigenvalues while $\tilde{\lambda}^{(1)}$ and $\tilde{\lambda}^{(3)}$ are left-eigenvalues.

\subsection{Conservation laws}

We now turn our attention to the time dependence of the solution $\psi(x, t, \lambda)$ of the pair of equations (9) and (10), and of the spectral data, to the purpose of finding quantities which do not depend on time. Let us consider first the continuum spectrum (real $\lambda$ ) and the solutions $\Psi_{L}(x, t, \lambda)$ and $\Psi_{R}(x, t, \lambda)$ which satisfy the boundary conditions (22). These conditions imply that the evolution equations (10) holds

$$
\Psi_{L t}=i \lambda\left[B, \Psi_{L}\right]+F(x, t) \Psi_{L}, \Psi_{R t}=i \lambda\left[B, \Psi_{R}\right]+F(x, t) \Psi_{R}
$$

therefore (see (25)) the evolution equation of the scattering matrix $S(\lambda, t)$ is

$$
S_{t}=i \lambda[B, S]
$$

The evolution equation of the scattering matrix $\tilde{S}(\lambda, t)$ is similarly found to be

$$
\tilde{S}_{t}=-i \lambda[B, \tilde{S}]
$$

These evolution equations, (48) and (49), clearly imply that the diagonal elements of $S(\lambda, t)$ and $\tilde{S}(\lambda, t)$ are indeed constants of the motion, and in particular

$S_{11}(\lambda, t)=S_{11}(\lambda), S_{33}(\lambda, t)=S_{33}(\lambda), \tilde{S}_{11}(\lambda, t)=\tilde{S}_{11}(\lambda), \tilde{S}_{33}(\lambda, t)=\tilde{S}_{33}(\lambda)$

We now consider the way to derive constants of the motion which are expressed in terms of the wave fields $u_{n}(x, t)$ and $v_{n}(x, t)$ rather than of scattering matrix 
elements. The relation to start with comes from the integral expressions (33) and (35) as taken in the limit $x \rightarrow+\infty$

$$
\begin{gathered}
S(\lambda, t)=\mathbf{1}+\int_{-\infty}^{+\infty} d x e^{-i \lambda A x} E(x, t) \Phi_{L}(x, t, \lambda) e^{i \lambda A x} \\
\tilde{S}(\lambda, t)=\mathbf{1}-\int_{-\infty}^{+\infty} d x e^{i \lambda A x} E^{T}(x, t) \tilde{\Phi}_{L}(x, t, \lambda) e^{-i \lambda A x} .
\end{gathered}
$$

Next we observe that the matrices $\Phi_{L}(x, t, \lambda)$ and $\tilde{\Phi}_{L}(x, t, \lambda)$ possess (f.i. see $[20])$ the following asymptotic expansion as $|\lambda|$ is very large

$$
\begin{aligned}
& \Phi_{L}(x, t, \lambda)=\mathbf{1}+\left(\frac{1}{\lambda}\right) \Phi_{L}^{(1)}(x, t)+\left(\frac{1}{\lambda}\right)^{2} \Phi_{L}^{(2)}(x, t)+\cdots, \\
& \tilde{\Phi}_{L}(x, t, \lambda)=\mathbf{1}+\left(\frac{1}{\lambda}\right) \tilde{\Phi}_{L}^{(1)}(x, t)+\left(\frac{1}{\lambda}\right)^{2} \tilde{\Phi}_{L}^{(2)}(x, t)+\cdots,
\end{aligned}
$$

which, when inserted in the integral relations (51) and (52) for the diagonal entries, yields the corresponding asymptotic expansions

$$
\begin{aligned}
& S_{11}(\lambda)=1+\left(\frac{1}{\lambda}\right) S_{11}^{(1)}+\left(\frac{1}{\lambda}\right)^{2} S_{11}^{(2)}+\cdots, \\
& S_{33}(\lambda)=1+\left(\frac{1}{\lambda}\right) S_{33}^{(1)}+\left(\frac{1}{\lambda}\right)^{2} S_{33}^{(2)}+\cdots,
\end{aligned}
$$

and, analogously,

$$
\begin{aligned}
& \tilde{S}_{11}(\lambda)=1+\left(\frac{1}{\lambda}\right) \tilde{S}_{11}^{(1)}+\left(\frac{1}{\lambda}\right)^{2} \tilde{S}_{11}^{(2)}+\cdots, \\
& \tilde{S}_{33}(\lambda)=1+\left(\frac{1}{\lambda}\right) \tilde{S}_{33}^{(1)}+\left(\frac{1}{\lambda}\right)^{2} \tilde{S}_{33}^{(2)}+\cdots .
\end{aligned}
$$

The computation of the asymptotic coefficients $S_{11}^{(n)}, S_{33}^{(n)}, \tilde{S}_{11}^{(n)}, \tilde{S}_{33}^{(n)}$ goes via the computation of the coefficients $\Phi_{L}^{(n)}(x, t)$ and $\tilde{\Phi}_{L}^{(n)}(x, t)$ of the matrix asymptotic expansions (53) whose expression can be recursively obtained by inserting the expansions (53) in the ODE satisfied by the matrices $\Phi_{L}(x, t, \lambda)$ and $\tilde{\Phi}_{L}(x, t, \lambda)$, namely

$$
\begin{gathered}
\Phi_{L x}=i \lambda\left[A, \Phi_{L}\right]+E(x) \Phi_{L}, \\
\tilde{\Phi}_{L x}=-i \lambda\left[A, \tilde{\Phi}_{L}\right]-E^{T}(x) \tilde{\Phi}_{L},
\end{gathered}
$$

with the appropriate boundary value $\Phi_{L}(x, t, \lambda) \rightarrow \mathbf{1}$ and $\tilde{\Phi}_{L}(x, t, \lambda) \rightarrow \mathbf{1}$ as $x \rightarrow-\infty$. This procedure yields an infinite number of constants of the motion as functionals of the wave fields. Here we limit ourselves to derive their explicit expression only for $n=1$ and $n=2$. While we omit detailing these computations as they are straight, though rather tedious, the explicit expressions we find are

$$
S_{11}^{(1)}=i \int_{-\infty}^{+\infty} d x\left(\frac{u_{2} v_{2}}{a_{3}-a_{1}}+\frac{u_{3} v_{3}}{a_{2}-a_{1}}\right), S_{33}^{(1)}=i \int_{-\infty}^{+\infty} d x\left(\frac{u_{1} v_{1}}{a_{2}-a_{3}}+\frac{u_{2} v_{2}}{a_{1}-a_{3}}\right),
$$




$$
\begin{gathered}
\tilde{S}_{11}^{(1)}=-S_{11}^{(1)}, \quad \tilde{S}_{33}^{(1)}=-S_{33}^{(1)} \\
S_{11}^{(2)}=\int_{-\infty}^{+\infty} d x\left[\frac{u_{2 x} v_{2}}{\left(a_{3}-a_{1}\right)^{2}}+\frac{u_{3} v_{3 x}}{\left(a_{2}-a_{1}\right)^{2}}+\frac{\left(u_{1} u_{2} u_{3}+v_{1} v_{2} v_{3}\right)}{\left(a_{1}-a_{2}\right)\left(a_{3}-a_{1}\right)}\right]+\frac{1}{2} S_{11}^{(1) 2}, \\
S_{33}^{(2)}=\int_{-\infty}^{+\infty} d x\left[\frac{u_{1 x} v_{1}}{\left(a_{3}-a_{2}\right)^{2}}+\frac{u_{2} v_{2 x}}{\left(a_{3}-a_{1}\right)^{2}}+\frac{\left(u_{1} u_{2} u_{3}+v_{1} v_{2} v_{3}\right)}{\left(a_{3}-a_{1}\right)\left(a_{2}-a_{3}\right)}\right]+\frac{1}{2} S_{33}^{(1) 2}, \\
\tilde{S}_{11}^{(2)}=\int_{-\infty}^{+\infty} d x\left[\frac{u_{2} v_{2 x}}{\left(a_{3}-a_{1}\right)^{2}}+\frac{u_{3 x} v_{3}}{\left(a_{2}-a_{1}\right)^{2}}-\frac{\left(u_{1} u_{2} u_{3}+v_{1} v_{2} v_{3}\right)}{\left(a_{1}-a_{2}\right)\left(a_{3}-a_{1}\right)}\right]+\frac{1}{2} \tilde{S}_{11}^{(1) 2}, \\
\tilde{S}_{33}^{(2)}=\int_{-\infty}^{+\infty} d x\left[\frac{u_{1} v_{1 x}}{\left(a_{3}-a_{2}\right)^{2}}+\frac{u_{2 x} v_{2}}{\left(a_{3}-a_{1}\right)^{2}}-\frac{\left(u_{1} u_{2} u_{3}+v_{1} v_{2} v_{3}\right)}{\left(a_{3}-a_{1}\right)\left(a_{2}-a_{3}\right)}\right]+\frac{1}{2} \tilde{S}_{33}^{(1) 2} .
\end{gathered}
$$

We conclude therefore that computing the first two coefficients of the expansions (54) and (55) leads to the four conserved quantities $S_{11}^{(1)}, S_{33}^{(1)}, S_{11}^{(2)}, S_{33}^{(2)}$ because of (57b) and because the formulae (58), together with (57b), imply the relations $S_{11}^{(2)}+\tilde{S}_{11}^{(2)}=S_{11}^{(1) 2}$ and $S_{33}^{(2)}+\tilde{S}_{33}^{(2)}=S_{33}^{(1) 2}$.

Consider next the discrete spectrum. As a consequence of (50) the discrete eigenvalues, which are the zeros of the time-independent functions $S_{11}(\lambda)$, $S_{33}(\lambda), \tilde{S}_{11}(\lambda), \tilde{S}_{33}(\lambda)$, are also constants of the motion, and therefore the evolution of both the spectral equations (18) and its adjoint (19) is isospectral.

\subsection{Reduction to the $3 W R I$ model}

Here we reduce the system (8) of six PDEs for the fields variables $u_{n}, v_{n}$ to the three coupled equations (2) of the resonant interaction model for the three fields $E_{n}$. To this purpose we consider the following relations

$$
\begin{aligned}
& u_{n}=\frac{\eta_{n}}{w^{\frac{3}{2}}} \sqrt{(-1)^{n}\left(V_{n+1}-V_{n+2}\right)} E_{n}, \\
& v_{n}=(-1)^{n+1} \frac{\eta}{w^{\frac{3}{2}}} \sqrt{(-1)^{n}\left(V_{n+1}-V_{n+2}\right)} E_{n}^{*}, n=1,2,3, \bmod 3,
\end{aligned}
$$

where $\eta=\eta_{1} \eta_{2} \eta_{3}$, see $(7)$, and

$$
w^{3}=\left(V_{3}-V_{1}\right)\left(V_{2}-V_{1}\right)\left(V_{3}-V_{2}\right)>0 .
$$

As a consequence, the matrix $E(x, t)$, see (16), which takes the expression

$$
E=\frac{1}{w^{\frac{3}{2}}}\left(\begin{array}{lcr}
0 & \eta_{3} \sqrt{V_{2}-V_{1}} E_{3} & -\eta \sqrt{V_{3}-V_{1}} E_{2}^{*} \\
\eta \sqrt{V_{2}-V_{1}} E_{3}^{*} & 0 & \eta_{1} \sqrt{V_{3}-V_{2}} E_{1} \\
\eta_{2} \sqrt{V_{3}-V_{1}} E_{2} & \eta \sqrt{V_{3}-V_{2}} E_{1}^{*} & 0
\end{array}\right)
$$

satisfies the reduction condition (a dagger stands for Hermitian conjugation)

$$
E^{\dagger}=-U E U^{-1},
$$


where $U$ is the constant, real and diagonal matrix

$$
U=\left(\begin{array}{ccc}
\eta_{1} & 0 & 0 \\
0 & -\eta_{2} & 0 \\
0 & 0 & \eta_{3}
\end{array}\right) \quad, \quad U^{2}=\mathbb{I}
$$

The straight implication of this property (62) is that, for any fundamental solution $\Psi(x, t, \lambda)$ of the spectral equation (18), also the matrix $\Psi^{\prime}(x, t, \lambda)=$ $U^{-1} \Psi^{\dagger-1}\left(x, t, \lambda^{*}\right)$ is a fundamental solution. This proposition yields the reduction condition for the particular fundamental solutions $\Psi_{L}(x, t, \lambda)$ and $\Psi_{R}(x, t, \lambda)$, i. e.

$$
\Psi_{L}(x, t, \lambda)=U^{-1} \Psi_{L}^{\dagger-1}\left(x, t, \lambda^{*}\right) U, \quad \Psi_{R}(x, t, \lambda)=U^{-1} \Psi_{R}^{\dagger-1}\left(x, t, \lambda^{*}\right) U,
$$

which are written here under the assumption, for the sake of simplicity, that the matrix $E(x, t)(61)$ is defined on a compact support of the $x$-axis so that the solutions $\Psi(x, t, \lambda)$ of (18) are entire functions of the spectral variable $\lambda$. However the results reported below apply as well under weaker assumptions. The scattering relation (25), if combined with (64), gives the reduction condition (unitary relation)

$$
S(\lambda)=U^{-1} S\left(\lambda^{*}\right)^{\dagger-1} U
$$

on the scattering matrix and, therefore, the relation

$$
S(\lambda)=U^{-1} \tilde{S}^{*}\left(\lambda^{*}\right) U
$$

between the scattering matrices $S(\lambda)$ and $\tilde{S}(\lambda)$. On the continuum spectrum $\lambda=\lambda^{*}$, this condition yields the relation $S(\lambda)=U^{-1} \tilde{S}^{*}(\lambda) U$ which, for matrix elements, reads

$$
\tilde{S}_{j n}(\lambda)=\frac{U_{j j}}{U_{n n}} S_{j n}^{*}(\lambda)=(-1)^{j+n} \eta_{j} \eta_{n} S_{j n}^{*}(\lambda) .
$$

For future reference we note that this formula, together with (29), leads to the unitarity relations

$$
\begin{aligned}
& \left|S_{11}\right|^{2}-\eta_{1} \eta_{2}\left|S_{12}\right|^{2}+\eta_{1} \eta_{3}\left|S_{13}\right|^{2}=1, \\
& \left|S_{11}\right|^{2}-\eta_{1} \eta_{2}\left|S_{21}\right|^{2}+\eta_{1} \eta_{3}\left|S_{31}\right|^{2}=1, \\
& \left|S_{33}\right|^{2}+\eta_{1} \eta_{3}\left|S_{31}\right|^{2}-\eta_{2} \eta_{3}\left|S_{32}\right|^{2}=1, \\
& \left|S_{33}\right|^{2}+\eta_{1} \eta_{3}\left|S_{13}\right|^{2}-\eta_{2} \eta_{3}\left|S_{23}\right|^{2}=1 .
\end{aligned}
$$

Next we consider the reduction conditions on the discrete spectrum. From (67) we infer that

$$
S_{11}(\lambda)=\tilde{S}_{11}^{*}\left(\lambda^{*}\right), \quad S_{33}(\lambda)=\tilde{S}_{33}^{*}\left(\lambda^{*}\right)
$$


which readily implies, by comparing (40) with (42) and (41) with (39), that the discrete eigenvalues come in pair of complex conjugates, i. e.

$$
\lambda^{(1)}=\tilde{\lambda}^{(1) *}, \lambda^{(3)}=\tilde{\lambda}^{(3) *} .
$$

Therefore the computation of the discrete eigenvalues is confined to computing the zeros of $S_{11}(\lambda)$ in the upper half-plane and of $S_{33}(\lambda)$ in the lower half-plane so that the spectrum is the collection of the eigenvalues $\left\{\lambda^{(1)}, \lambda^{(3) *}\right\}$ in the upper half-plane and $\left\{\lambda^{(3)}, \lambda^{(1) *}\right\}$ in the lower half-plane. Moreover, according to the general scheme of subsection 3.2, to each of these eigenvalues there corresponds one eigenfunction, precisely $\chi^{(1)}$ and $\chi^{(3)}$ to the left-eigenvalues $\lambda^{(1)}$ and, respectively, $\lambda^{(3)}$, and $\tilde{\chi}^{(1)}$ and $\tilde{\chi}^{(3)}$ to the right-eigenvalues $\lambda^{(1) *}$ and, respectively, $\lambda^{(3) *}$.

We finally turn our attention to the conservation laws of the 3WRI model, and to their spectral decomposition, namely to their expression in terms of contributions coming from the continuous and discrete parts of the spectrum. In order to derive this decomposition, we make use of the analyticity properties of the diagonal entries $S_{11}(\lambda)$, and $S_{33}(\lambda)$ we have established in subsection 3.2. Here we follow the treatment applied to the Korteweg-de Vries equation in [33]. We start by observing that the functions $\sigma_{1}(\lambda)$ and $\sigma_{3}(\lambda)$, defined as

$$
\begin{aligned}
& \sigma_{1}(\lambda)=\ln \left[S_{11}(\lambda) \prod_{n}\left(\frac{\lambda-\lambda_{n}^{(+1) *}}{\lambda-\lambda_{n}^{(+1)}}\right)\right], \\
& \sigma_{3}(\lambda)=\ln \left[S_{33}^{*}\left(\lambda^{*}\right) \prod_{n}\left(\frac{\lambda-\lambda_{n}^{(-3)}}{\lambda-\lambda_{n}^{(-3) *}}\right)\right.
\end{aligned}
$$

are analytic in the upper-half plane $\operatorname{Im} \lambda>0$ and vanish as $O(1 / \lambda)$ or faster if $\lambda \rightarrow \infty$. A standard application of the Cauchy theorem to a closed contour composed of the real $\lambda$-axis and of a semicircle at infinity in the upper half plane yields the integral representation

$$
\sigma_{j}(\lambda)=\frac{1}{i \pi} P \int_{-\infty}^{+\infty} d \mu \frac{\sigma_{j}(\mu)}{\mu-\lambda}, \operatorname{Im} \lambda=0, j=1,3
$$

where $\mathrm{P}$ denotes the principal value. To our present purpose it is convenient to consider only the imaginary part of this relation (72), together with the unitarity relations (68), to obtain the formulae

$$
\begin{array}{r}
\operatorname{Im} \sigma_{1}(\lambda)=-\frac{1}{2 \pi} P \int_{-\infty}^{+\infty} \frac{d \mu}{\mu-\lambda} \ln \left[1+\eta_{1} \eta_{2}\left|S_{12}\right|^{2}\right. \\
\left.-\eta_{1} \eta_{3}\left|S_{13}\right|^{2}\right], \operatorname{Im} \lambda=0, \\
\operatorname{Im} \sigma_{3}(\lambda)=-\frac{1}{2 \pi} P \int_{-\infty}^{+\infty} \frac{d \mu}{\mu-\lambda} \ln \left[1-\eta_{1} \eta_{3}\left|S_{13}\right|^{2}\right. \\
\left.+\eta_{2} \eta_{3}\left|S_{23}\right|^{2}\right], \operatorname{Im} \lambda=0 .
\end{array}
$$


At this point we perform the large $\lambda$ asymptotic expansion of both sides of these equations by taking into account (54) and by noticing that, with appropriate differentiability conditions on the matrix $E(x, t)$ with respect to the variable $x$, the off-diagonal elements of the scattering matrix $S(\lambda)$ vanish, as $\lambda \rightarrow \infty$, faster than any power of $\lambda$. By limiting the expansion to the order $\mathrm{O}(1 / \lambda)$ and $\mathrm{O}\left(1 / \lambda^{2}\right)$ terms only, we obtain

$$
\begin{aligned}
\operatorname{Im}\left(S_{11}^{(1)}+2 \sum_{n} \lambda_{n}^{(+1)}\right)= & \frac{1}{2 \pi} \int_{-\infty}^{+\infty} d \lambda \ln \left[1+\eta_{1} \eta_{2}\left|S_{12}\right|^{2}\right. \\
& \left.-\eta_{1} \eta_{3}\left|S_{13}\right|^{2}\right], \\
\operatorname{Im}\left(S_{33}^{(1)}+2 \sum_{n} \lambda_{n}^{(-3)}\right)= & -\frac{1}{2 \pi} \int_{-\infty}^{+\infty} d \lambda \ln \left[1-\eta_{1} \eta_{3}\left|S_{13}\right|^{2}\right. \\
& \left.+\eta_{2} \eta_{3}\left|S_{23}\right|^{2}\right], \\
\operatorname{Im}\left(S_{11}^{(2)}+\sum_{n} \lambda_{n}^{(+1) 2}-\frac{1}{2} S_{11}^{(1) 2}\right)= & \frac{1}{2 \pi} \int_{-\infty}^{+\infty} d \lambda \lambda \ln \left[1+\eta_{1} \eta_{2}\left|S_{12}\right|^{2}\right. \\
& \left.-\eta_{1} \eta_{3}\left|S_{13}\right|^{2}\right], \\
\operatorname{Im}\left(S_{33}^{(2)}+\sum_{n} \lambda_{n}^{(-3) 2}-\frac{1}{2} S_{33}^{(1) 2}\right)= & -\frac{1}{2 \pi} \int_{-\infty}^{+\infty} d \lambda \lambda \ln \left[1-\eta_{1} \eta_{3}\left|S_{13}\right|^{2}\right. \\
& \left.+\eta_{2} \eta_{3}\left|S_{23}\right|^{2}\right] .
\end{aligned}
$$

The expressions $(57 \mathrm{a}, 58 \mathrm{a}, 58 \mathrm{~b})$ of the asymptotic coefficients $S_{11}^{(1)}, S_{33}^{(1)}, S_{11}^{(2)}, S_{33}^{(2)}$, if specialised to the 3WRI reduction (59), become

$$
S_{11}^{(1)}=\frac{i \eta}{3 w^{3}} M_{1}, \quad S_{33}^{(1)}=\frac{i \eta}{3 w^{3}} M_{3}
$$

where the constants of the motion $M_{1}$ and $M_{3}$ are the Manley-Rowe invariants

$$
M_{1}=\int_{-\infty}^{+\infty} d x\left(\eta_{3}\left|E_{3}\right|^{2}-\eta_{2}\left|E_{2}\right|^{2}\right), M_{3}=\int_{-\infty}^{+\infty} d x\left(\eta_{2}\left|E_{2}\right|^{2}-\eta_{1}\left|E_{1}\right|^{2}\right),
$$

and

$$
S_{11}^{(2)}=\frac{i \eta}{9 w^{3}} H_{1}+\frac{1}{2} S_{11}^{(1) 2}, S_{33}^{(2)}=\frac{i \eta}{9 w^{3}} H_{3}+\frac{1}{2} S_{33}^{(1) 2},
$$

where the constants of the motion $H_{1}$ and $H_{3}$ are defined as

$$
\begin{aligned}
& H_{1}=\int_{-\infty}^{+\infty} d x \operatorname{Im}\left[\frac{\eta_{3}}{V_{1}-V_{2}} E_{3}^{*} E_{3 x}-\frac{\eta_{2}}{V_{3}-V_{1}} E_{2}^{*} E_{2 x}+\frac{2}{\left(V_{1}-V_{2}\right)\left(V_{3}-V_{1}\right)} E_{1} E_{2} E_{3}\right], \\
& H_{3}=\int_{-\infty}^{+\infty} d x \operatorname{Im}\left[\frac{\eta_{2}}{V_{3}-V_{1}} E_{2}^{*} E_{2 x}-\frac{\eta_{1}}{V_{2}-V_{3}} E_{1}^{*} E_{1 x}+\frac{2}{\left(V_{2}-V_{3}\right)\left(V_{3}-V_{1}\right)} E_{1} E_{2} E_{3}\right] .
\end{aligned}
$$

Therefore we finally obtain the spectral decomposition of the four conserved quantities $M_{1}, M_{3}, H_{1}, H_{3}$ which reads, for the Manley-Rowe invariants,

$$
\begin{aligned}
M_{1}=3 \eta w^{3} & \left\{\frac { 1 } { 2 \pi } \int _ { - \infty } ^ { + \infty } d \lambda \operatorname { l n } \left[1+\eta_{1} \eta_{2}\left|S_{12}\right|^{2}\right.\right. \\
& \left.\left.-\eta_{1} \eta_{3}\left|S_{13}\right|^{2}\right]-2 \sum_{n} \operatorname{Im} \lambda_{n}^{(+1)}\right\},
\end{aligned}
$$




$$
\begin{aligned}
M_{3}=3 \eta w^{3} & \left\{-\frac{1}{2 \pi} \int_{-\infty}^{+\infty} d \lambda \ln \left[1-\eta_{1} \eta_{3}\left|S_{13}\right|^{2}\right.\right. \\
& \left.\left.+\eta_{2} \eta_{3}\left|S_{23}\right|^{2}\right]-2 \sum_{n} \operatorname{Im} \lambda_{n}^{(-3)}\right\},
\end{aligned}
$$

and for the other two constants of the motion

$$
\begin{aligned}
H_{1}=9 \eta w^{3} & \left\{\frac { 1 } { 2 \pi } \int _ { - \infty } ^ { + \infty } d \lambda \lambda \operatorname { l n } \left[1+\eta_{1} \eta_{2}\left|S_{12}\right|^{2}\right.\right. \\
& \left.\left.-\eta_{1} \eta_{3}\left|S_{13}\right|^{2}\right]-\sum_{n} \operatorname{Im}\left(\lambda_{n}^{(+1) 2}\right)\right\}, \\
H_{3}=9 \eta w^{3} & \left\{-\frac{1}{2 \pi} \int_{-\infty}^{+\infty} d \lambda \lambda \ln \left[1-\eta_{1} \eta_{3}\left|S_{13}\right|^{2}\right.\right. \\
& \left.\left.+\eta_{2} \eta_{3}\left|S_{23}\right|^{2}\right]-\sum_{n} \operatorname{Im}\left(\lambda_{n}^{(-3) 2}\right)\right\} .
\end{aligned}
$$

We observe that these formulae provide a neat expression of conserved quantities of the 3WRI model in terms of the discrete eigenvalues and of the offdiagonal entries of the scattering matrix on the continuum spectrum. These last spectral data can be numerically computed in the perspective of the direct spectral problem by integrating the spectral differential equation (9) for a fixed, arbitrary value of the time variable $t$ (see below). However, in the standard approach to the inverse spectral problem (see [20]) the spectral data which are relevant to the reconstruction of the matrix $E(x, t)$ are instead the so-called reflection coefficients. Though it is easy to derive also a decomposition formula for the constants of the motion (76) and (78) in terms of reflection coefficients, we omit this derivation here as we find our formulae (79) and (80) more convenient to our purpose of numerical computation.

As we are concerned with numerical computations, we deem it convenient to provide analytic explicit solutions of the direct problem to the purpose of testing numerical methods and codes. Thus we end this section by reporting such expressions for the one-soliton solution [18] which may be obtained by means of Darboux transformations as given in [34-36]. This solution of the 3WRI equations (2) reads

$$
\begin{gathered}
E_{1}(x, t)=\frac{-6 \eta_{2} p w^{3 / 2}\left(V_{3}-V_{2}\right)^{1 / 2} A_{2} A_{3}^{*} e^{3(p-i q)\left(V_{3}-V_{2}\right)\left(x-V_{1} t\right)}}{\left|A_{1}\right|^{2} e^{-6 p\left(V_{2}-V_{1}\right)\left(x-V_{3} t\right)}-\eta_{1} \eta_{2}\left|A_{2}\right|^{2}+\eta_{1} \eta_{3}\left|A_{3}\right|^{2} e^{6 p\left(V_{3}-V_{2}\right)\left(x-V_{1} t\right)}} \\
E_{2}(x, t)=\frac{6 \eta p w^{3 / 2}\left(V_{3}-V_{1}\right)^{1 / 2} A_{3} A_{1}^{*} e^{3(p+i q)\left(V_{3}-V_{1}\right)\left(x-V_{2} t\right)}}{\left|A_{1}\right|^{2}-\eta_{1} \eta_{2}\left|A_{2}\right|^{2} e^{6 p\left(V_{2}-V_{1}\right)\left(x-V_{3} t\right)}+\eta_{1} \eta_{3}\left|A_{3}\right|^{2} e^{6 p\left(V_{3}-V_{1}\right)\left(x-V_{2} t\right)}} \\
E_{3}(x, t)=\frac{6 \eta_{3} p w^{3 / 2}\left(V_{2}-V_{1}\right)^{1 / 2} A_{1} A_{2}^{*} e^{3(p-i q)\left(V_{2}-V_{1}\right)\left(x-V_{3} t\right)}}{\left|A_{1}\right|^{2}-\eta_{1} \eta_{2}\left|A_{2}\right|^{2} e^{6 p\left(V_{2}-V_{1}\right)\left(x-V_{3} t\right)}+\eta_{1} \eta_{3}\left|A_{3}\right|^{2} e^{6 p\left(V_{3}-V_{1}\right)\left(x-V_{2} t\right)}}
\end{gathered}
$$

and depends on the two real parameters $q$ and $p$ and on the three complex parameters $A_{1}, A_{2}, A_{3}$. All other symbols are well defined in this subsection. This solution is in the BBB class and is regular (i.e. everywhere bounded) only if $\eta_{1} \eta_{2}=-1$ and $\eta_{1} \eta_{3}=1$, namely in the soliton exchange case. We shortly illustrate here this solution by looking at its asymptotic behavior as $t \rightarrow \pm \infty$. If this limit is taken in the $(x, t)$ plane with $x=V t+y$, and $y$ is a fixed spacial co-ordinate, then, if $V$ is different from any one of the three 
characteristic velocities, $V \neq V_{n}, n=1,2,3$, then all three fields exponentially vanish, $E_{n}(V t+y, t) \rightarrow 0$, when $t \rightarrow \pm \infty$. If instead $V$ coincides with one of the velocities $V_{n}$, then $E_{n}\left(V_{m} t+y, t\right), n, m=1,2,3$, also vanishes as $t \rightarrow$ $\pm \infty$ with the following three exceptions: $E_{1}\left(V_{1} t+y, t\right) \rightarrow E_{1}^{(-)}(y), E_{3}\left(V_{3} t+\right.$ $y, t) \rightarrow E_{3}^{(-)}(y)$ as $t \rightarrow-\infty$, and $E_{2}\left(V_{2} t+y, t\right) \rightarrow E_{2}^{(+)}(y)$ as $t \rightarrow+\infty$. The three asymptotic profiles $E_{1}^{(-)}(y), E_{2}^{(+)}(y), E_{3}^{(-)}(y)$ have the standard envelope shape

$$
\begin{gathered}
E_{1}^{(-)}(y)=\frac{-3 \eta_{2} p w^{3 / 2}\left(V_{3}-V_{2}\right)^{1 / 2} e^{i \phi_{1}} e^{-3 i q\left(V_{3}-V_{2}\right) y}}{\cosh \left[3 p\left(V_{3}-V_{2}\right)\left(y-y_{1}\right)\right]} \\
E_{2}^{(+)}(y)=\frac{3 \eta p w^{3 / 2}\left(V_{3}-V_{1}\right)^{1 / 2} e^{i \phi_{2}} e^{3 i q\left(V_{3}-V_{1}\right) y}}{\cosh \left[3 p\left(V_{3}-V_{1}\right)\left(y-y_{2}\right)\right]} \\
E_{3}^{(-)}(y)=\frac{3 \eta_{3} p w^{3 / 2}\left(V_{2}-V_{1}\right)^{1 / 2} e^{i \phi_{3}} e^{-3 i q\left(V_{2}-V_{1}\right) y}}{\cosh \left[3 p\left(V_{2}-V_{1}\right)\left(y-y_{3}\right)\right]}
\end{gathered}
$$

where $\phi_{1}+\phi_{2}+\phi_{3}=0$. Here we have set, with no loss of generality,

$$
A_{1}=e^{\frac{3}{2} p\left(V_{3}-V_{1}\right) y_{2}}, A_{2}=e^{\frac{3}{2} p\left[\left(V_{3}-V_{2}\right) y_{1}-\left(V_{2}-V_{1}\right) y_{3}\right]-i \phi_{3}}, A_{3}=e^{-\frac{3}{2} p\left(V_{3}-V_{1}\right) y_{2}+i \phi_{2}} .
$$

Thus this solution describes the collision of the two initial pulses $E_{1}^{(-)}\left(x-V_{1} t\right)$ and $E_{3}^{(-)}\left(x-V_{3} t\right)$ which, after collision, merge into the single pulse $E_{2}^{(+)}(x-$ $V_{2} t$ ) (which turns out to be unstable, see below). The co-ordinates $y_{1}$ and $y_{3}$ provide the positions of the incoming pulses while $y_{2}$, which refers to the position of the outgoing pulse, turns out to be predicted, via the previous formulae (83), by the relation

$$
y_{2}=\frac{\left(V_{3}-V_{2}\right) y_{1}+\left(V_{2}-V_{1}\right) y_{3}}{\left(V_{3}-V_{1}\right)}
$$

We also note that the time reverted process, which is obtained from this solution (81) via the time reversal transformation $x \rightarrow-x, t \rightarrow-t, E_{n} \rightarrow$ $-E_{n}$, describes instead the decay of the input pulse $-E_{2}^{(+)}\left(-x+V_{2} t\right)$ into the two pulses $-E_{1}^{(-)}\left(-x+V_{1} t\right)$ and $-E_{3}^{(-)}\left(-x+V_{3} t\right)$.

Let us consider now the eigenvalue and eigenfunction associated with this soliton solution. We note first that the Darboux transformation [35] yields a fundamental solution of the Lax pair of equations (9) and (10) whose explicit expression is

$$
\Psi(x, t, \lambda)=\left[\mathbf{1}+\left(\frac{\alpha-\alpha^{*}}{\lambda-\alpha}\right) \frac{\zeta \zeta^{\dagger} \Sigma}{\zeta^{\dagger} \Sigma \zeta}\right] e^{i \lambda(A x+B t)}
$$

where the complex number $\alpha$ is related to the soliton parameters $q, p$ (see (81)) as $\alpha=q+i p$. Here we have introduced the diagonal sign matrix $\Sigma=\operatorname{diag}$ 
$\left\{1,-\eta_{1} \eta_{2}, \eta_{1} \eta_{3}\right\}$ and the vector

$$
\zeta=\zeta(x, t)=e^{i \alpha^{*}(A x+B t)} \mu, \mu=\left(\begin{array}{c}
\eta_{1} A_{1} \\
\eta_{2} A_{2} \\
\eta_{3} A_{3}
\end{array}\right)
$$

where the complex parameters $A_{n}$ are those as in the soliton solution (81). Here and in the following, we assume that the parameter $p$ be positive, $p>0$; the opposite case $p<0$ can be similarly treated. On the continuum spectrum, namely for real values of $\lambda$, the expression of the special solutions $\Psi_{L}(x, t, \lambda)$ and $\Psi_{R}(x, t, \lambda)$ follows from (85) and reads

$$
\begin{aligned}
& \Psi_{L}(x, t, \lambda)=\left[\mathbf{1}+\left(\frac{\alpha-\alpha^{*}}{\lambda-\alpha}\right) \frac{\zeta \zeta^{\dagger} \Sigma}{\zeta^{\dagger} \Sigma \zeta}\right]\left[\mathbf{1}-\left(\frac{\alpha-\alpha^{*}}{\lambda-\alpha^{*}}\right) P_{1}\right] e^{i \lambda A x}, \\
& \Psi_{R}(x, t, \lambda)=\left[\mathbf{1}+\left(\frac{\alpha-\alpha^{*}}{\lambda-\alpha}\right) \frac{\zeta \zeta^{\dagger} \Sigma}{\zeta^{\dagger} \Sigma \zeta}\right]\left[\mathbf{1}-\left(\frac{\alpha-\alpha^{*}}{\lambda-\alpha^{*}}\right) P_{3}\right] e^{i \lambda A x},
\end{aligned}
$$

where $P_{1}$ is the diagonal projector matrix $P_{1}=\operatorname{diag}\{1,0,0\}$ and $P_{3}$ is the diagonal projector matrix $P_{3}=\operatorname{diag}\{0,0,1\}$. Thus these solutions imply that the scattering matrix $S(\lambda)$ is diagonal,

$$
S_{n m}(\lambda)=S_{n n}(\lambda) \delta_{n m}, S_{11}=\left(\frac{\lambda-\alpha}{\lambda-\alpha^{*}}\right), S_{22}=1, S_{33}=\left(\frac{\lambda-\alpha^{*}}{\lambda-\alpha}\right),
$$

and that, therefore, the reflection coefficients are vanishing. The discrete eigenvalues, since they coincide with the zeros of $S_{11}(\lambda)$ in the upper half-plane $\operatorname{Im} \lambda>0$ and with the zeros of $S_{33}(\lambda)$ in the lower half-plane $\operatorname{Im} \lambda<0$, are $\lambda^{(1)}=\alpha$ and $\lambda^{(3)}=\alpha^{*}$ (see (40) and (41)). Thus there are only two eigenvalues and, because of the peculiar property $S_{33}(\lambda)=S_{11}^{*}\left(\lambda^{*}\right)$ of this one-soliton solution, two eigenfunctions correspond to the same eigenvalue, as they are solutions of the equation (30) for different values of the parameter $a$. Precisely the eigenfunctions $\chi^{(1)}, \tilde{\chi}^{(3)}$ correspond to the eigenvalue $\alpha$ and $\tilde{\chi}^{(1)}, \chi^{(3)}$ correspond to the eigenvalue $\alpha^{*}$. Their expression, as derived by applying the recipe given in $(39),(40),(41)$ and $(42)$, reads

$$
\begin{gathered}
\chi^{(1)}(x, t)=\eta_{1} A_{1}^{*} e^{-i \alpha\left[\left(\frac{a_{1}+a_{2}}{2}\right) x+b_{1} t\right]} \frac{\zeta(x, t)}{\zeta^{\dagger}(x, t) \Sigma \zeta(x, t)}, \\
\tilde{\chi}^{(3)}(x, t)=\eta_{1} A_{3}^{*} e^{-i \alpha\left[\left(\frac{a_{3}+a_{2}}{2}\right) x+b_{3} t\right]} \frac{\zeta(x, t)}{\zeta^{\dagger}(x, t) \Sigma \zeta(x, t)}, \\
\tilde{\chi}^{(1)}(x, t)=e^{i \alpha^{*}\left(\frac{a_{1}-a_{2}}{2}\right) x}\left[\left(\begin{array}{l}
1 \\
0 \\
0
\end{array}\right)-\eta_{1} A_{1}^{*} e^{-i \alpha\left(a_{1} x+b_{1} t\right)} \frac{\zeta(x, t)}{\zeta^{\dagger}(x, t) \Sigma \zeta(x, t)}\right],
\end{gathered}
$$




$$
\chi^{(3)}(x, t)=e^{i \alpha^{*}\left(\frac{a_{3}-a_{2}}{2}\right) x}\left[\left(\begin{array}{l}
0 \\
0 \\
1
\end{array}\right)-\eta_{1} A_{3}^{*} e^{-i \alpha\left(a_{3} x+b_{3} t\right)} \frac{\zeta(x, t)}{\zeta^{\dagger}(x, t) \Sigma \zeta(x, t)}\right] .
$$

As for the regularity condition for the soliton solution (81), these eigenfunctions are $L_{2}$ functions only in the soliton exchange case $\eta_{1} \eta_{2}=-1$ and $\eta_{1} \eta_{3}=1$.

\section{BBD waves}

This section is devoted to those solutions of the 3WRI equations (2) which satisfy the boundary conditions (3) and (5), and describe therefore the interaction of two bright pulses, $E_{1}$ and $E_{2}$, and one, $E_{3}$, which is instead a pulse which travels on a background. This background is a plane wave so that the condition (5) is better specified by the asymptotic expressions

$$
E_{3}(x, t) \rightarrow E_{3}^{( \pm)}(x, t), x \rightarrow \pm \infty
$$

where the background plane-waves at $x= \pm \infty$ have same wave-number and amplitude but possibly different phase, namely

$$
E_{3}^{( \pm)}(x, t)=\frac{w^{\frac{3}{2}}}{\sqrt{V_{2}-V_{1}}} c e^{2 i\left[k\left(x-V_{3} t\right)+\theta_{ \pm}\right]}
$$

The class of solutions which satisfy these conditions is therefore characterized by the parameters $2 k$, the wave-number, $c$, the amplitude (the pre-factor is merely due to convenience) and two different phases, $2 \theta_{+}$and $2 \theta_{-}$. Similarly to the case of $\mathrm{BBB}$ waves discussed in the previous section, our focus now is on the spectral properties of the solutions $\psi(x, \lambda)$ of the differential matrix equation (18) at fixed time, f.i. at $t=0$. Moreover, since our interest is on the decay and exchange of solitons, we confine our discussion to the 3WRI reduction of subsection 3.4 with the particular choice of signs $\eta_{1}=\eta_{3}=1$ and $\eta_{2}=-1$. Thus the matrix $E(x)$ (see (61))

$$
E=\frac{1}{w^{\frac{3}{2}}}\left(\begin{array}{ccr}
0 & \sqrt{V_{2}-V_{1}} E_{3} & \sqrt{V_{3}-V_{1}} E_{2}^{*} \\
-\sqrt{V_{2}-V_{1}} E_{3}^{*} & 0 & \sqrt{V_{3}-V_{2}} E_{1} \\
-\sqrt{V_{3}-V_{1}} E_{2}-\sqrt{V_{3}-V_{2}} E_{1}^{*} & 0
\end{array}\right),
$$

in the equation (18) is anti-Hermitian and takes the asymptotic expression (see (91) and (92)

$$
E(x) \rightarrow E^{( \pm)}(x), x \rightarrow \pm \infty
$$


with

$$
E^{( \pm)}(x)=W^{( \pm)}(x)\left(\begin{array}{lll}
0 & c & 0 \\
-c & 0 & 0 \\
0 & 0 & 0
\end{array}\right) W^{( \pm)-1}(x) .
$$

where we have set

$$
W^{( \pm)}(x)=\left(\begin{array}{ccc}
e^{i\left(k x+\theta_{ \pm}\right)} & 0 & 0 \\
0 & e^{-i\left(k x+\theta_{ \pm}\right)} & 0 \\
0 & 0 & 1
\end{array}\right)
$$

The spectral methods in this case require the construction of fundamental solutions $\Psi^{( \pm)}(x, \lambda)$ of the differential equation (18) corresponding to the asymptotic expressions (95), namely

$$
\Psi_{x}^{( \pm)}=\left[i \lambda A+E^{( \pm)}(x)\right] \Psi^{( \pm)}
$$

For any complex value of the spectral variable $\lambda$ these solutions are found to be

$$
\Psi^{( \pm)}(x, \lambda)=\left(\begin{array}{lcr}
e^{i\left(w_{1}+k\right) x} & i \mu e^{i\left[\left(w_{2}+k\right) x+2 \theta_{ \pm}\right]} & 0 \\
i \mu e^{i\left[\left(w_{1}-k\right) x-2 \theta_{ \pm}\right]} & e^{i\left(w_{2}-k\right) x} & 0 \\
0 & 0 & e^{i \lambda a_{3} x}
\end{array}\right),
$$

with the following definitions:

$$
\begin{aligned}
& w_{1}=\sigma \lambda+\sqrt{(\delta \lambda-k)^{2}+c^{2}}, \quad w_{2}=\sigma \lambda-\sqrt{(\delta \lambda-k)^{2}+c^{2}}, \\
& \mu=\frac{c}{\delta \lambda-k+\sqrt{(\delta \lambda-k)^{2}+c^{2}}}, \quad \sigma=\frac{1}{2}\left(a_{1}+a_{2}\right), \delta=\frac{1}{2}\left(a_{1}-a_{2}\right) .
\end{aligned}
$$

We note that the expression (98) is so chosen to coincide in the BBB limit $c \rightarrow 0, k \rightarrow 0$ with the $\operatorname{expression} \exp (i \lambda x A)$ used in the previous section, see $(22)$.

If $\lambda$ is real, in analogy with the $\mathrm{BBB}$ case, we define the matrix solutions $\Psi_{L}(x, \lambda)$ and $\Psi_{R}(x, \lambda)$ by the asymptotic behaviour

$$
\begin{aligned}
& \Psi_{L}(x, \lambda) \longrightarrow \Psi^{(-)}(x, \lambda) \quad, \quad x \rightarrow-\infty, \\
& \Psi_{R}(x, \lambda) \longrightarrow \Psi^{(+)}(x, \lambda) \quad, \quad x \rightarrow+\infty,
\end{aligned}
$$

and the scattering matrix $S(\lambda)$ via the standard way $\Psi_{L}=\Psi_{R} S(\lambda)$. This definition implies that also in the present case $\operatorname{det} S(\lambda)=1$. We do not go into any detail here and we merely notice that, since the "wave-numbers" $w_{1}(\lambda), w_{2}(\lambda)$ are real for real $\lambda$, the real values of $\lambda$ belong to the continuum spectrum since any solution of (18) is bounded and oscillating. 
To our purpose of numerical computation and applications to soliton dynamics, we point out that BBB solutions and BBD solutions differ from each other with respect to their propagation. In fact, while the BBB pulses locally interact with each other and then eventually propagate without dispersion, the two brights in the BBD triplet continuously and forever interact with the background with the consequence that they propagate with dispersion. In this respect soliton solutions, like simultons and boomerons, see [27,28,30], acquire special importance. The computational scheme to numerically find discrete eigenvalues associated to solitons in the BBD case parallels the one we have described in the previous section, and is briefly reported here to pinpoint technical differences. Again a complex number $\lambda$ is a discrete eigenvalue of the spectral equation (18) if a real number $a$ exists such that the ODE (30) possesses a vector solution in $L_{2}(-\infty,+\infty)$. The way to ascertain wether a vector solution $\psi(x, \lambda)$ is in $L_{2}$ is by looking at its exponential behavior at large $|x|$, which is

$$
\begin{aligned}
& \psi(x, \lambda) \rightarrow \Psi^{( \pm)}(x, \lambda)\left(\begin{array}{c}
c_{ \pm}^{(1)}(\lambda) \\
c_{ \pm}^{(2)}(\lambda) \\
c_{ \pm}^{(3)}(\lambda)
\end{array}\right) \\
& =\left(\begin{array}{c}
c_{ \pm}^{(1)}(\lambda) e^{i x\left(w_{1}(\lambda)+k+a \lambda\right)}+i \mu(\lambda) c_{ \pm}^{(2)}(\lambda) e^{i\left[x\left(w_{2}(\lambda)+k+a \lambda\right)+2 \theta_{ \pm}\right]} \\
i \mu(\lambda) c_{ \pm}^{(1)}(\lambda) e^{i\left[x\left(w_{1}(\lambda)-k+a \lambda\right)-2 \theta_{ \pm}\right]}+c_{ \pm}^{(2)}(\lambda) e^{i x\left(w_{2}(\lambda)-k+a \lambda\right)} \\
c_{ \pm}^{(3)}(\lambda) e^{i \lambda x\left(a_{3}+a\right)}
\end{array}\right), x \rightarrow \pm \infty
\end{aligned}
$$

where the vector $\left(c_{ \pm}^{(1)}, c_{ \pm}^{(2)}, c_{ \pm}^{(3)}\right)$ is $x$-independent and the three functions $w_{1}(\lambda), w_{2}(\lambda), \mu(\lambda)$ are defined by the explicit expressions (99). In order to proceed further we note that also in the present case the statement that, if $\lambda$ is an eigenvalue, then also its complex conjugate $\lambda^{*}$ belong to the discrete spectrum, holds true. This property of the spectrum allows one to look only for eigenvalues which lie in the upper plane, say for $\operatorname{Im} \lambda>0$. Moreover, we fix the sign of the square root $\sqrt{\left[(\delta \lambda-k)^{2}+c^{2}\right]}$ in (99) by asking that $\operatorname{Im} \sqrt{\left[(\delta \lambda-k)^{2}+c^{2}\right]}<0$ so that, in the BBB limit $c \rightarrow 0, k \rightarrow 0$, one has $w_{1} \rightarrow a_{1} \lambda$ and $w_{2} \rightarrow a_{2} \lambda$. It is now important to notice that, with these specifications, the ordering (15) together with the vanishing of the trace of $A$ (see (11)) imply the ordering relation

$$
\operatorname{Im} w_{1}<\operatorname{Im} w_{2}<a_{3} \operatorname{Im} \lambda
$$

whose proof is simple and is not reported here. We are now in the position to repeat the argument given in subsection 3.2 to provide the following step-bystep computational method:

(1) choose $a=-\frac{1}{2}\left(\operatorname{Im} w_{2}+a_{3} \operatorname{Im} \lambda\right)$ and integrate (30) from $x=+\infty$ by 
assuming the initial conditions $c_{1}^{(+)}=c_{2}^{(+)}=0$ and $c_{3}^{(+)}=1$,

(2) compute $c_{3}^{(-)}(\lambda)$,

(3) find the zeros $\lambda^{(+)}$(if any) of $c_{3}^{(-)}(\lambda)$, say $c_{3}^{(-)}\left(\lambda^{(+)}\right)=0$,

(4) choose $a=-\frac{1}{2}\left(\operatorname{Im} w_{1}+\operatorname{Im} w_{2}\right)$ and integrate (30) from $x=-\infty$ by assuming the initial conditions $c_{2}^{(-)}=c_{3}^{(-)}=0$ and $c_{1}^{(-)}=1$,

(5) compute $c_{1}^{(+)}(\lambda)$,

(6) find the zeros $\lambda^{(+)}$(if any) of $c_{1}^{(+)}(\lambda)$, say $c_{1}^{(+)}\left(\lambda^{(+)}\right)=0$.

These zeros $\lambda^{(+)}$(if any) together with their complex conjugates are in the discrete spectrum.

We close this section by noticing that also in the BBD class of solutions the discrete eigenvalues are time-independent. Therefore the computation of eigenvalues can be done at any fixed time. As implied by the boundary conditions (91) and (92) it is sufficient to replace the coordinate $x$ with the characteristic coordinate $x-V_{3} t$ which, for fixed time, does not affect the asymptotic exponential behavior of the eigenfunctions and the computational method.

\section{$5 \quad$ Numerical results}

In this section we discuss few examples of wave profiles with the purpose of applying the computational algorithms introduced in sections 3 and 4 , and to test their accuracy. Hereafter we consider the 3WRI equations (2) with $\left(\eta_{1}, \eta_{2}, \eta_{3}\right)=(+,-,+)$, namely only the the soliton exchange type interaction since this is the case relevant to recently found results [24-32]. With no loss of generality, we shall use coordinates $(x, t)$ such that $V_{3}=0$. Owing to the property (70), the search for discrete eigenvalues only needs to be performed in the upper-half of the complex plane. This search leads to a discrete set of eigenvalues $\left\{\lambda_{n}^{(1)}\right\}$ and $\left\{\lambda_{m}^{(3) *}\right\}$ for the left and right spectrum, respectively.

\subsection{BBB solutions}

Let us consider first the BBB case. As a first example, we analyse the ZakharovManakov (ZM) soliton [18] (81). The soliton spectrum is known exactly: it consists of a discrete eigenvalue $\lambda=\alpha=q+i p$ in the upper half of the complex plane (i.e. $p>0$ so that its complex conjugate $\alpha^{*}$ lies in the lower half plane). On the continuum spectrum, there is no radiation, namely the reflection coefficients are vanishing, with the implication that the scattering matrix $S(\lambda)$ is diagonal (see (88)).

In the following we provide an example of the accuracy of the numerical method for computing spectral data associated with the ZM soliton. In Fig. 1 

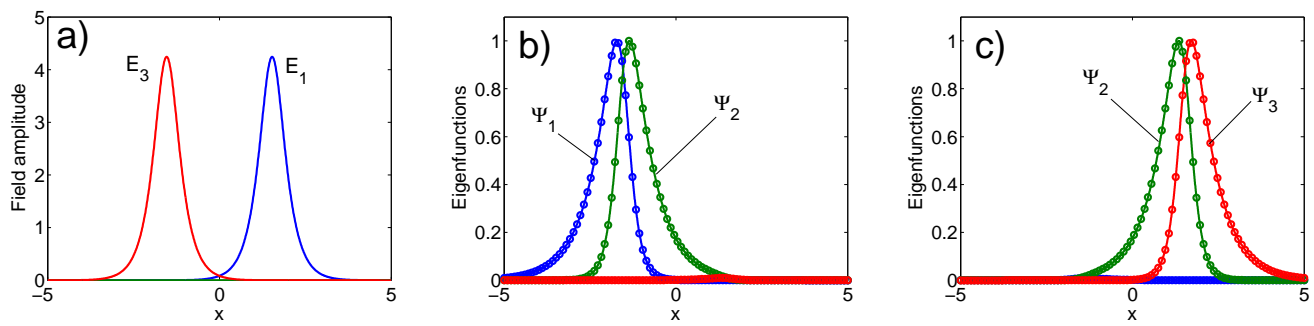

Fig. 1. (a) Field amplitudes $\left|E_{1}\right|,\left|E_{3}\right|$ of the ZM soliton with parameters $t=0$, $A_{1}=0.01, A_{2}=1, A_{3}=0.01, q=0.5, p=1$. The characteristic velocities are $V_{1}=-2, V_{2}=-1, V_{3}=0$. (b) Modulus of the eigenfunctions for the left spectrum $\left(a=-\frac{1}{2}\left(a_{1}+a_{2}\right)\right.$, eigenvalue $\lambda^{(1)}=0.4999999999956+0.99999999814319 i$, error $\left.\left|\alpha-\lambda^{(1)}\right|=1.86 \cdot 10^{-9}\right)$. (c) Modulus of the eigenfunctions for the right spectrum $\left(a=-\frac{1}{2}\left(a_{2}+a_{3}\right)\right.$, eigenvalue $\lambda^{(3) *}=0.4999999999956+0.99999999814319 i$, error $\left.\left|\alpha-\lambda^{(3) *}\right|=1.86 \cdot 10^{-9}\right)$. Solid curves and circles stand for numerical and exact analytical eigenfunctions, respectively.
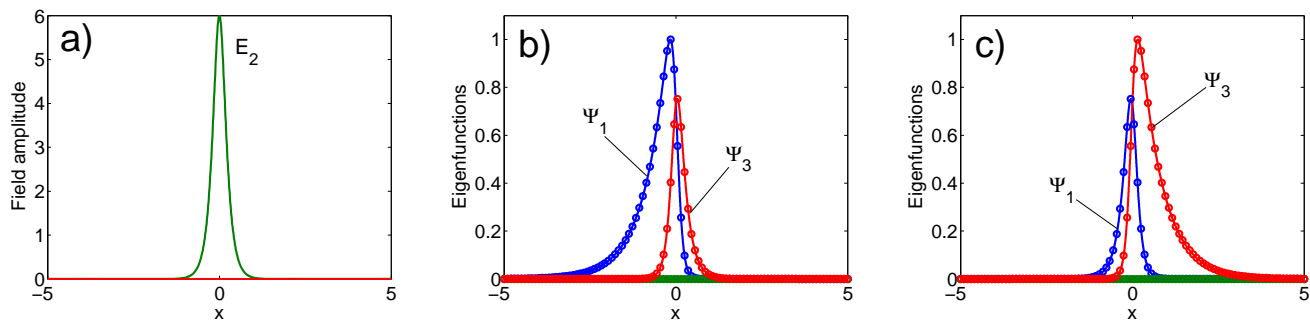

Fig. 2. (a) Field amplitude $\left|E_{2}\right|$ of the ZM soliton with parameters $t=0, A_{1}=1$, $A_{2}=0, A_{3}=1, q=0.5, p=1$. The characteristic velocities are $V_{1}=-2$, $V_{2}=-1, V_{3}=0$. (b) Modulus of the eigenfunctions for the left spectrum $\left(a=-\frac{1}{2}\left(a_{1}+a_{2}\right)\right.$, eigenvalue $\lambda^{(1)}=0.50000000001558+0.99999999992954 i$, error $\left.\left|\alpha-\lambda^{(1)}\right|=7.22 \cdot 10^{-11}\right)$. (c) Modulus of the eigenfunctions for the right spectrum $\left(a=-\frac{1}{2}\left(a_{2}+a_{3}\right)\right.$, eigenvalue $\lambda^{(3) *}=0.50000000001558+0.99999999992954$, error $\left.\left|\alpha-\lambda^{(3) *}\right|=7.22 \cdot 10^{-11}\right)$. Solid curves and circles stand for numerical and exact analytical eigenfunctions, respectively.

we illustrate the eigenfunctions that are associated with the ZM soliton components in waves $E_{1}$ and $E_{3}$. This case corresponds to the excitation of two bright pulses, and the spectrum is computed right before their collision. The exact discrete eigenvalue is equal to $\alpha=0.5+i$. The present numerical algorithm is able to compute this eigenvalue with a precision of $o\left(10^{-9}\right)$. Moreover we find that $\alpha$ is indeed a double eigenvalue, since it is present both in the right and left spectrum: $\lambda^{(1)}=\lambda^{(3) *}=\alpha$.

Panel 1(a) shows the field amplitudes $\left|E_{1}\right|$ and $\left|E_{3}\right|$ (as a matter of fact, a nonzero component of $E_{2}$ is also present but it is not visible on the scale of the figure). In panels 1(b) and 1(c) we plot with solid lines the eigenfunctions $\psi_{1}, \psi_{2}, \psi_{3}$ which are associated to the left and right spectrum, respectively. For the left spectrum, the eigenfunctions $\psi_{1}$ and $\psi_{2}$ which are localized around the position of the pulse $E_{3}$ : wave $E_{1}$ does not influence the shape of these eigen- 

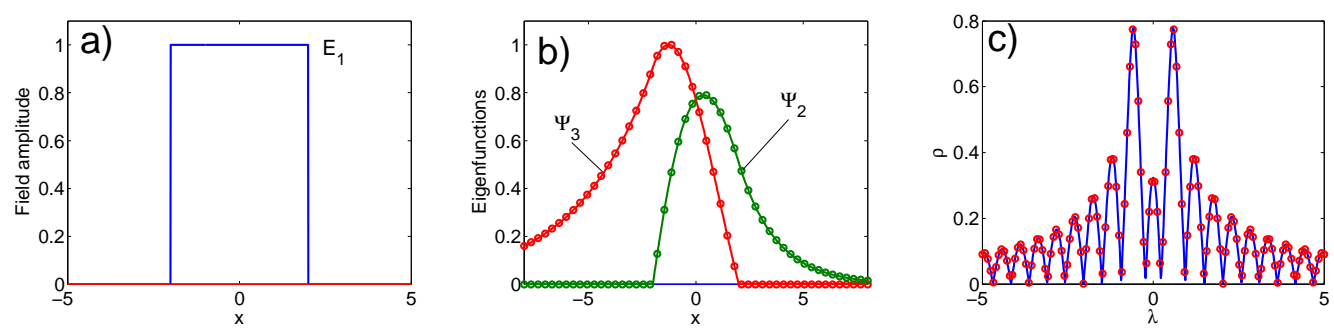

Fig. 3. (a) Rectangular pulse in wave $E_{1}$ with parameters $A=1, B=2$. The characteristic velocities are $V_{1}=-2, V_{2}=-1, V_{3}=0$. (b) Numerical (solid lines) and analytical (circles) (modulus of) eigenfunctions for the right spectrum $\left(a=V_{1}\right)$. (c) Numerical (solid lines) and analytical (circles) (modulus of) the reflection coefficient (104) on the continuum spectrum.

functions. The situation is just the opposite if we consider the right spectrum (see panel 1(c)). In the panels (b) and (c) the circles represent the analytical eigenfunctions (89), which turn out to be perfectly superimposed to the numerically calculated eigenfunctions. Inspection of the eigenfunctions and of their associated eigenvalues reveals that wave $E_{3}$ carries the discrete eigenvalue $\lambda^{(1)}$, whereas the wave $E_{1}$ carries the discrete eigenvalue $\lambda^{(3) *}$.

In Fig. 2 we illustrate the case of a ZM soliton where a relatively large wave $E_{2}$ results from the collision of the waves $E_{1}$ and $E_{3}$ (see $(82 \mathrm{~b})$ ). In the first panel 2(a) we plot the field amplitude $\left|E_{2}\right|$ as a function of $x$. In panels 2(b) and $2(\mathrm{c})$ we plot the modulus of the eigenfunctions associated to the left and right spectrum, respectively: both eigenfunctions are localized around the support of wave $E_{2}$. Again the left and right spectrum contains the same discrete eigenvalue $\alpha=0.5+i$. In this case the numerically computed eigenvalues are obtained with precision of $o\left(10^{-10}\right)$. The eigenfunctions corresponding to the right and left spectrum differ from each other, however in both cases nonzero $\psi_{1}$ and $\psi_{3}$ are obtained. In panels $2(\mathrm{~b})$ and 2(c) the circles represent the analytical eigenfunctions (89), which again turn out to be superimposed to the numerically calculated values (solid curves). In this case it can be seen that the wave $E_{2}$ carries both the discrete eigenvalues $\lambda^{(1)}$ and $\lambda^{(3) *}$.

To further assess the accuracy of the numerical computation of the direct spectral transform, we computed the spectral data on the continuum spectrum of the ZM soliton. Such solution has analytical reflection coefficients, or, equivalently, off-diagonal entries of the scattering matrix $S(\lambda)$, which are identically equal to zero, see (88). The computational noise associated with the numerical method yields nonzero but relatively small reflection coefficients. We found that for all real values of the spectral parameter $\lambda$ the numerically computed values of the reflection coefficients are $o\left(10^{-5}\right)$, which corresponds to the error tolerance that we used when solving the linear system (9).

In order to check the accuracy of the present numerical computational method in the case of non vanishing spectral data on the continuum spectrum, we consider a single rectangular pulse in wave $E_{1}$ and no pulse in $E_{2}$ and $E_{3}$. Such 
configuration leads to a straightforward analytical expression for the entire spectrum. Indeed, the direct scattering problem reduces to the standard $2 \times 2$ Zakharov-Shabat problem for the right spectrum ( the left spectrum is empty). The analytical discrete eigenvalues are the solutions of the transcendental equation

$$
\tan (s B)=\frac{i s}{3 \lambda\left(V_{3}-V_{2}\right)},
$$

in the lower half-plane $\operatorname{Im} \lambda<0$, where $s=\sqrt{4 c^{2}+9 \lambda^{2}\left(V_{3}-V_{2}\right)^{2}}, c=$ $A / \sqrt{\left(V_{3}-V_{1}\right)\left(V_{2}-V_{1}\right)}$, with $A$ and $2 B$ the amplitude and width of the rectangular pulse, respectively. The reflection coefficient on the continuum spectrum is

$$
\rho(\lambda)=\frac{S_{32}(\lambda)}{S_{22}(\lambda)}=-2 i c \frac{\sin (s B) e^{3 i \lambda\left(V_{2}-V_{3}\right) B}}{3 \lambda\left(V_{3}-V_{2}\right) \sin (s B)+i s \cos (s B)} .
$$

The panel (a) of Fig. 3 shows the rectangular envelope profile of the wave $E_{1}$ as a function of $x$. In this case there is a single discrete eigenvalue $\lambda=\lambda^{(3) *}$, plus a certain amount of radiation. The discrete eigenvalue is found to be $\lambda^{(3) *}=0.28952 i$ from (103), whereas the numerical computation yields $\lambda^{(3) *}=$ $-4.5416 \cdot 10^{-17}+0.28952 i$. Panel 3(b) compares the analytical (circles) and the numerically evaluated (continuous curves) amplitudes of the eigenfunctions: as it can be seen, no difference is noticeable on the scale of the figure. Moreover panel 3(c) compares the analytical (circles) with the numerical (solid line) reflection coefficient on the continuum spectrum: again an excellent quantitative agreement is observed. Note that the shape of the continuum spectrum in 3(c) reveals (when compared with a sinc function which is the linear or Fourier spectrum of the rectangular pulse) that the radiation component of the spectrum has given up some of its energy in the low $\lambda$ region. Such energy is carried by the discrete eigenvalue which is associated to the soliton content of the field. In other words, the inspection of panel 3(c) indicates that the soliton formation results at the expense of the low-wavenumber components of the continuum spectrum.

As a further check of the accuracy of numerical computation of the spectral transform, we calculated the Manley-Rowe invariants (76) (for a single wave in $E_{1}$ only $M_{3}$ is non zero) in the spectral domain (79). When calculated exactly in spatial domain, the invariant $M_{3}=-4$. We compared this analytical result with the computation of the integral of the scattering matrix elements over the spectral parameter range $\lambda=[-30,30]$ with 1000 point sampling. The result in the spectral domain is then evaluated as the sum of the contributions of the discrete spectrum and of radiation, and yields: $M_{3}=M_{3 s o l}+M_{3 r a d}=-0.5187-3.4743=-3.993$.

As a last example of application of the numerical spectral transform, we considered a general situation where all the three waves are nonvanishing and carry both a continuum and a discrete spectrum. We considered the three 

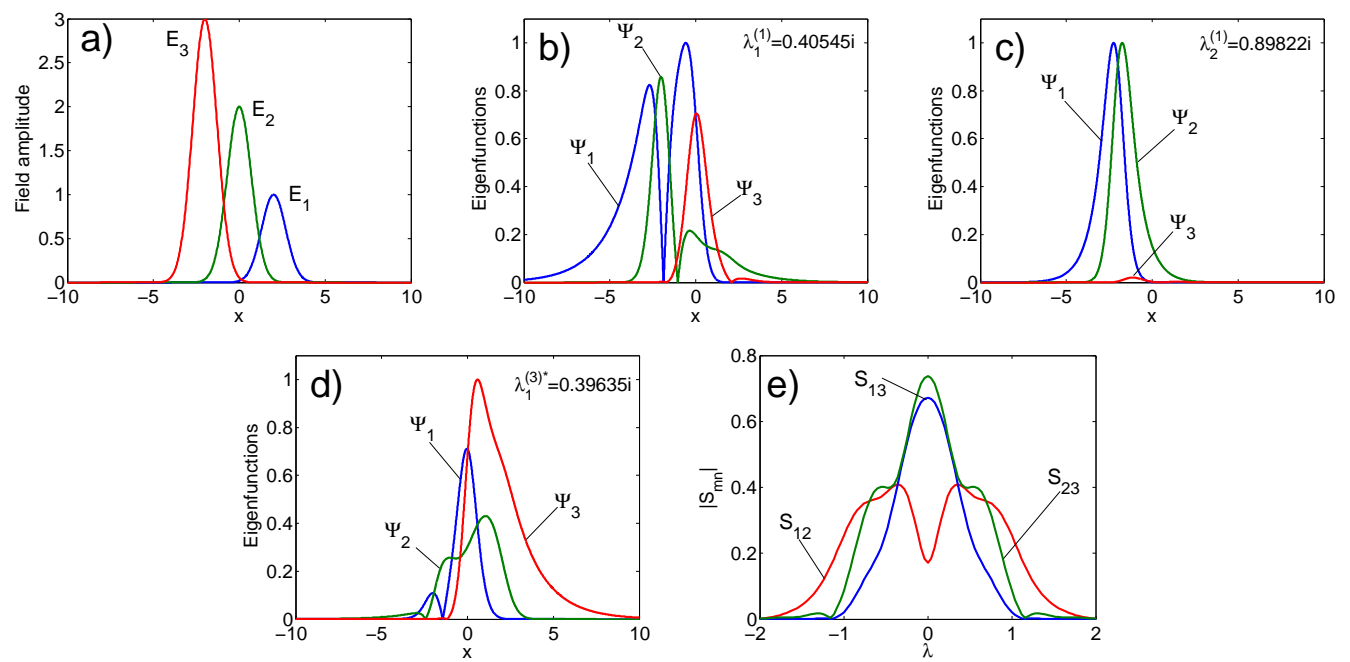

Fig. 4. (a) Field profiles of three gaussian pulses. The characteristic velocities are $V_{1}=-2, V_{2}=-1, V_{3}=0$. (b) Numerical (modulus of) eigenfunctions for the left eigenvalue $\lambda_{1}^{(1)}=0.4055 i$. (c) Numerical (modulus of) eigenfunctions for the left eigenvalue $\lambda_{2}^{(1)}=0.8992 i$. (d) Numerical (modulus of) eigenfunctions for the right eigenvalue $\lambda_{1}^{(3) *}=0.3964 i$. (e) Relevant entries of the scattering matrix $S$ over the continuum spectrum.

partially overlapping gaussian pulses: $E_{1}=\exp \left[-(x-2)^{2}\right], E_{2}=2 \exp \left[-x^{2}\right]$, $E_{3}=3 \exp \left[-(x+2)^{2}\right]$, whose profiles are plotted in Fig. 4(a). We found that the discrete left spectrum is composed by the two eigenvalues $\lambda_{1}^{(1)}=0.4055 i$ and $\lambda_{2}^{(1)}=0.8992 i$. The corresponding eigenfunctions are plotted in panels 4(b) and 4(c), respectively. From the computation of the scattering matrix elements $S_{12}$ and $S_{13}$, whose profile is shown in panel 4(e), we computed the invariant $M_{1}=M_{1 \text { sol } 1}+M_{1 \text { sol } 2}+M_{1 \text { rad }}=4.866+10.7786+0.6490=16.2934$, which agrees very well with the exact value $M_{1}=16.2931$ that is obtained from (76).

In this case we found that single eigenvalue exists in the right spectrum $\lambda_{1}^{(3) *}=$ $0.3964 i$ : the corresponding eigenfunction is shown in panel 4(d). From the computation of the scattering matrix elements $S_{13}$ and $S_{23}$ (see panel 4(e)) we calculated the invariant $M_{3}=M_{3 s o l 1}+M_{3 \mathrm{rad}}=-4.7562-1.5129=-6.2691$, that agrees reasonably well with the exact value $M_{3}=-6.2666$ that is obtained from (76).

\subsection{BBD solutions}

Let us consider next the accuracy of the numerical spectral transform method when applied to the BBD case. For such a test we used the boomeron soliton solution, whose analytical expression can be found in [28]. The boomeron is a soliton that describes the decay of an unstable simulton [27] (or velocity-locked 

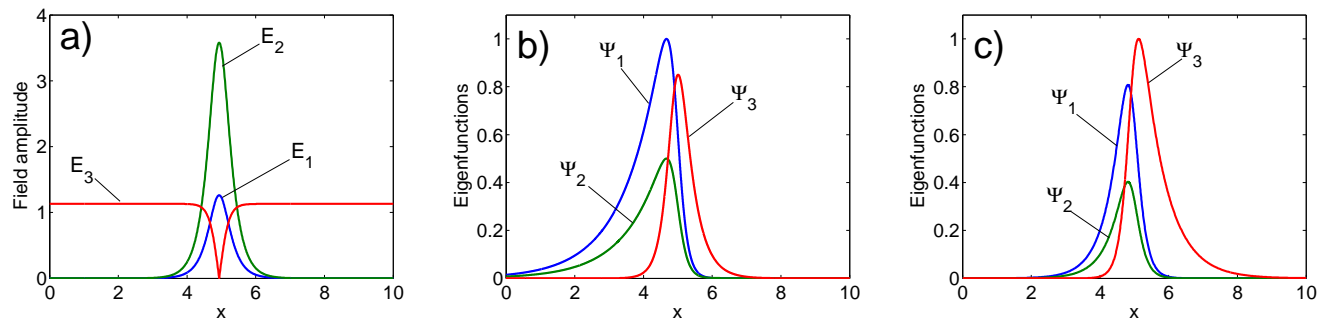

Fig. 5. (a) Boomeron amplitude profile [28] with parameters $p=-1, a=0.8$, $k=q=\theta=0$ at $t_{0}=-4.5$. The characteristic velocities are $V_{1}=-2$, $V_{2}=-1, V_{3}=0$. (b) Eigenfunctions for the left spectrum. Numerical eigenvalue $\lambda^{(1)}=0+0.66667 i$. (c) Eigenfunctions for the right spectrum. Numerical eigenvalue $\lambda^{(3) *}=3.8256 \cdot 10^{-17}+0.66667 i$.

bright-bright-dark triplet) into a stable simulton and an isolated single wave pulse, respectively. The boomeron is associated with a a discrete eigenvalue $\lambda=\frac{2}{3}(k+i p) /\left(V_{1}-V_{2}\right)$ and a zero continuum spectrum (see [28] for the details on the parameters $p, a, k, q, \theta)$.

Let us compare different boomeron field profiles to verify the precision of numerical direct spectral transform method. In Fig. 5 we show the case of a boomeron before its decay. In the first panel 5(a) we plot the field amplitudes $E_{1}, E_{2}, E_{3}$ of the simulton triplet as a function of $x$ for a given time $t_{0}$. In panels $5(\mathrm{~b})$ and $5(\mathrm{c})$ we report the numerically computed eigenfunctions that are associated with the left and right spectrum, respectively. Note that both left and right spectrum contain the same discrete eigenvalue $\lambda=2 / 3 i$ (such a value was numerically reproduced with a precision of $o\left(10^{-9}\right)$ ), even though the corresponding eigenfunctions are different in the two cases. Similarly to the case of the ZM soliton in Fig. 1, the presence of a double eigenvalue indicates that the three-wave fields in panel 5(a) indeed contains two separate solitons. As a result, the boomeron may decay through the emission of a relatively faster soliton (corresponding to the left spectrum) and a slower soliton (associated with the right spectrum).

Such situation indeed corresponds to the three wave field profiles illustrated in Fig. 6, which have been obtained after the boomeron decay. Panel 6(a) shows the (modulus of the) field amplitudes of the two separate solitons, namely a slower stable simulton triplet to the left and an isolated single soliton wave to the right. Again, in panels 6(b) and 6(c) we plotted by solid curves the (modulus of the) eigenfunctions that are associated with the left and the right spectrum, respectively. We numerically confirmed (with a precision of $o\left(10^{-9}\right)$ ) that the same discrete eigenvalue $\lambda=2 / 3 i$ is found in both the left and the right spectrum. From the plots of the spectral eigenfunctions in Fig. 6 we can see that the right spectrum is associated with the simulton triplet, whereas the left part of the spectrum is associated with the isolated wave in $E_{3}$.

It is interesting to point out that the application of the direct spectral transform technique permits to demonstrate the fully soliton nature of the single 

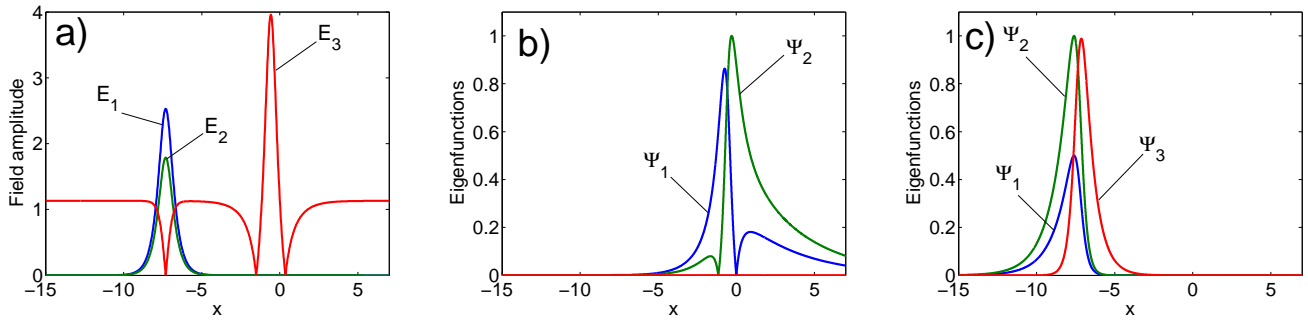

Fig. 6. (a) Boomeron amplitude profile [28] with parameters $p=-1, a=0.8$, $k=q=\theta=0$ at $t_{0}=4.5$. The characteristic velocities are $V_{1}=-2$, $V_{2}=-1, V_{3}=0$. (b) Eigenfunctions for the left spectrum. Numerical eigenvalue $\lambda^{(1)}=0+0.66667 i$ (c) Eigenfunctions for the right spectrum. Numerical eigenvalue $\lambda^{(3) *}=3.8256 \cdot 10^{-17}+0.66667 i$

wave in the $E_{3}$ component that appears to the right of panel 6(a). Such a wave is emitted after the decay of an unstable simulton triplet, a phenomenon that was extensively discussed in earlier work (see Refs. [27,28]). As last examples of application of the numerical implementation of the direct spectral transform method let us consider the interaction that results from the initial linear superposition of two identical but $x$-shifted stable simultons. In the absence of the other triplet, each simulton would propagate with the same velocity. This case is significant since for such an initial field profile the analytical spectrum is not known, hence the spectrum may only be computed numerically. The interaction of the pulse tails of the bright simulton components leads to either an attractive or repulsive force among the two simultons, depending upon their relative phases [28]. The panel (a) of Fig. 7 shows the numerical evolution in the $t-x$ plane of the component $E_{1}$ of an initial wave profile which is composed of two equal and in-phase stable simultons. In the absence of the other simulton, each triplet would travel with the same velocity $V=-1.7$ (the spectral eigenvalue of each individual simulton is equal to $\lambda=0.8744 i$ ). As shown in Fig. 7(a), the two triplets attract each other and their nonlinear superposition leads to a periodic breathing behavior. In panels $7(\mathrm{~b})$ and $7(\mathrm{c})$ we illustrate the two eigenfunctions which are both associated with the right spectrum. We show only the right spectrum here, since we found that the left spectrum does not contain any solitons. The numerically evaluated discrete spectrum exhibits two distinct complex eigenvalues with the same imaginary part and opposite real part $\left(\lambda_{1,2}^{(3) *}= \pm 0.11163+0.83319 i\right)$. Such a spectrum corresponds to two simulton solutions, both propagating with the same velocity $V=-1.685$ (the corresponding trajectory is plotted as a dashed line in panel (a) of Fig. 7).

On the other hand, panel (a) of Fig. 8 shows the numerically computed evolution in the $t-x$ plane of the component $E_{1}$ of an initial wave profile which is initially composed by the linear superposition of two equal and out-of-phase stable simultons. In the absence of the other triplet, each of these simultons would individually propagate with velocity $V=-1.7$. Fig. 8 (a) shows that the two triplets repel each other and their interaction leads to the generation 

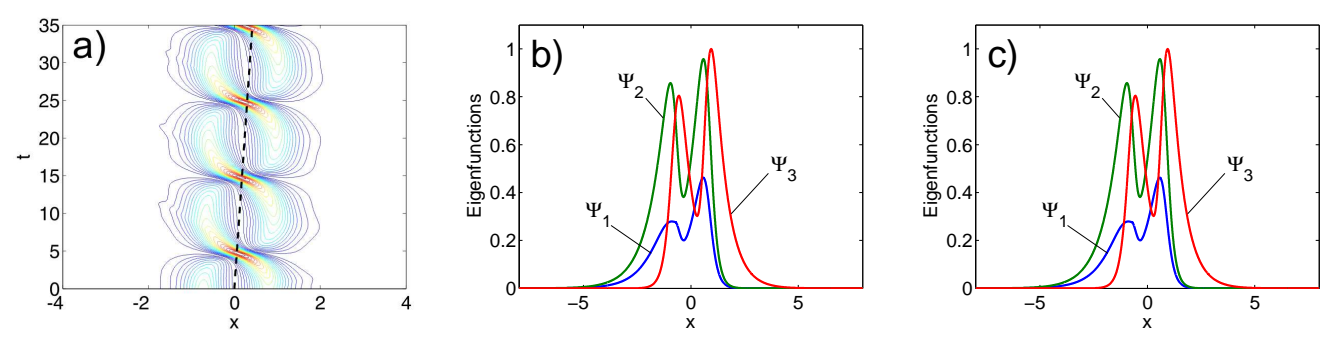

Fig. 7. (a) Spatio-temporal evolution of $E_{1}$ component. The initial wave profile is composed of two equal and in-phase stable simultons with velocity $V=-1.7$. The simulation is performed in a reference frame $V_{r e f}=V=-1.7$. (b) Eigenfunctions corresponding to eigenvalue $\lambda_{1}^{(3) *}=0.11163+0.83319 i$. (c) Eigenfunctions corresponding to eigenvalue $\lambda_{2}^{(3) *}=-0.11163+0.83319 i$.
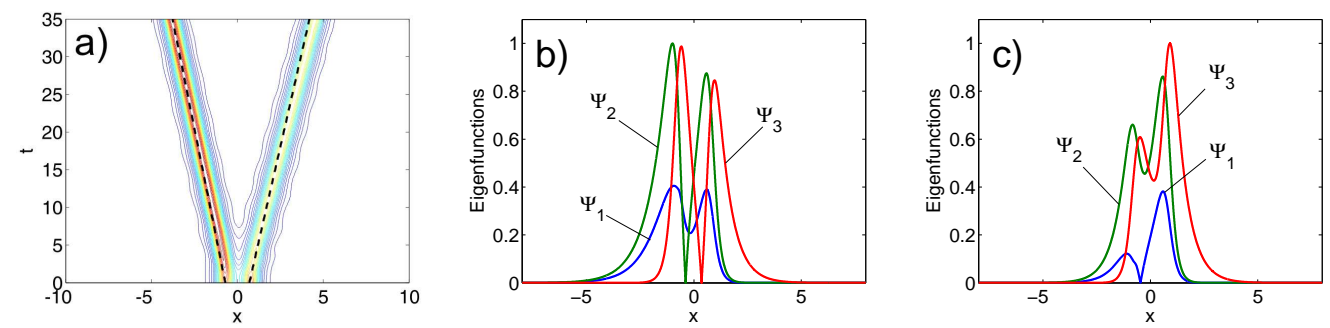

Fig. 8. (a) Spatio-temporal evolution of $E_{1}$ component. The initial wave profile is composed of two equal and out of phase stable simultons with velocity $V=-1.7$. The simulation is performed in a reference frame $V_{r e f}=V=-1.7$. (b) Eigenfunctions corresponding to eigenvalue $\lambda_{1}^{(3) *}=-6.8573 \cdot 10^{-17}+0.77151 i$. (c) Eigenfunctions corresponding to eigenvalue $\lambda_{2}^{(3) *}=-3.2111 \cdot 10^{-17}+1.0311 i$.

of two simultons that propagate with a different velocity. In panels (b) and (c) of Fig. 8 we illustrate the eigenfunctions that are associated with the right spectrum. The corresponding discrete spectrum consists of two distinct and purely imaginary eigenvalues $\lambda_{1}^{(3) *}=0.77151 i$ and $\lambda_{2}^{(3) *}=1.0311 i$, which involve two different simultons that propagate with velocities $V=-1.788$ and $V=-1.6021$, respectively. The trajectories of such simultons are shown as dashed lines in panel (a) of Fig. 8. Again we only show the right spectrum, since it turns out that the left spectrum does not contain any solitons.

\section{Conclusions}

We have developed numerical methods for computing the direct spectral problem for the three wave resonant interaction equations. We have introduced the spectral problem for both the discrete and the continuum parts of the spectrum when the boundary conditions were vanishing. We have also considered the spectral problem in the case the boundary conditions were non vanishing. We 
have included the numerical algorithms which have been designed to compute the discrete eigenvalues and also the reflection coefficients on the continuum spectrum. Then we have reported examples which illustrate the accuracy of the computational methods by comparing numerical with analytical results. The proposed direct spectral theory can be used to better understand and explain the physical structures of recent theoretical and experimental discoveries in the three-wave scenario [24-32].

\section{References}

[1] M.J. Ablowitz, D.J. Kaup, A.C. Newell and H. Segur, Method for Solving the Sine-Gordon Equation Phys. Rev. Lett. 30, 1262 (1973).

[2] C.S. Gardner, J.M. Greene, M.D. Kruskal and R.M. Miura, Method for Solving the Korteweg-deVries Equation Phys. Rev. Lett. 19, 1095 (1967).

[3] V.E. Zakharov and A.B. Shabat, Exact theory of two-dimensional self-focusing and one-dimensional self-modulation of waves in non linear media $\mathrm{Zh}$. Exp. Teor. Fiz., 61, 118 (1971) (Russian); English transl. in Sov. Phys. JEPT, 34, 62 (1972).

[4] M.J. Ablowitz and H. Segur, Solitons and the Inverse Scattering Transform (SIAM, Philadelphia, 1981).

[5] M. Wadati, J. Phys. Soc. Japan, The Exact Solution of the Modified Korteweg-de Vries Equation 32, 1681 (1972).

[6] F. Calogero and A. Degasperis, Solution by spectral-transform method of a nonlinear evolution equation including as a special case cylindrical KDV equation Lett. Nuovo Cim. 23, 150 (1978).

[7] A.V. Mikhailov, On the integrability of two-dimensional generalization of the toda Lattice Sov. Phys. JEPT Lett. 30, 414 (1979).

[8] A.P. Fordy and J. Gibbons, Integrable non-linear klein-gordon equations and toda-lattices Commun. Math. Phys. 77, 21 (1980).

[9] K. Pohlmeyer, Integrable hamiltonian systems and interactions through quadratic constraints Commun. Math. Phys. 46, 207 (1976).

[10] F. Lund and T. Regge, Unified approach to strings and vortices with soliton solutions Phys. Rev. D14, 1524 (1976).

[11] J.D. Gibbon, P.J. Caudrey, R.K. Bullough and J.C. Eilbeck, AnN-soliton solution of a nonlinear optics equation derived by a general inverse method Lett. Nuovo Cim. 8, 775 (1973).

[12] G.L. Lamb, Phase Variation in Coherent-Optical-Pulse Propagation Phys. Rev. Lett. 31, 196 (1973). 
[13] M.J. Ablowitz, D.J. Kaup and A.C. Newell, Coherent Pulse Propagation A Dispersive Irreversible Phenomenon J. Math. Phys. 15, 1852 (1974).

[14] S.V. Manakov, Complete integrability and stochastization of discrete dynamical systems Sov. Phys. JEPT 40, 269 (1975).

[15] A.V. Mikhailov, Integrability of 2-dimensional thirring model JEPT Lett. 23, 320 (1976).

[16] J. Villarroel, The DBAR problem and the thirring model Stud. Appl. Math. 84, 207 (1991).

[17] M. Kruskal, The Korteweg-de Vries equation and related evolution equations Lect. Appl. Math. 15, 61 (1974).

[18] V.E. Zakharov and S.V. Manakov, Resonance interaction of wave packets Sov. Phys. JEPT Lett. 18, 243 (1973).

[19] M.J. Ablowitz and R. Haberman, Resonantly Coupled Nonlinear Evolution Equations J. Math. Phys. 16, 2301 (1975).

[20] D.J. Kaup, The three-Wave Interaction-A Nondispersive Phenomenon Stud. Appl. Math 55, 9-44 (1976).

[21] M.J. Ablowitz and P.A. Clarkson, Solitons, Nonlinear Evolution Equations and Inverse Scattering (Cambridge University Press, 1991).

[22] A.C. Newell, Solitons in Mathematics and Physics (SIAM, Philadelphia, 1985).

[23] R.K. Dodd, J.C. Eilbeck, J.D. Gibbon, and H.C. Morris, Solitons and Nonlinear Wave Equations (Academic Press, London, 1982).

[24] A. Picozzi and M. Haelterman, Spontaneous formation of symbiotic solitary waves in a backward quasi-phase-matched parametric oscillator Opt. Lett. 23, 1808 (1998).

[25] V. M. Malkin, G. Shvets, and N. J. Fish, Detuned Raman Amplification of Short Laser Pulses in Plasma Phys. Rev. Lett. 84, 1208 (2000).

[26] I. Y. Dodin and N. J. Fish, Storing, Retrieving, and Processing Optical Information by Raman Backscattering in Plasmas Phys. Rev. Lett. 88, 165001 (2002).

[27] A. Degasperis, M. Conforti, F. Baronio and S. Wabnitz, Stable Control of Pulse Speed in Parametric Three-Wave Solitons Phys. Rev. Lett. 97, 093901 (2006).

[28] M. Conforti, A. Degasperis, F. Baronio and S. Wabnitz, Inelastic scattering and interactions of three-wave parametric solitons Phys. Rev. E 74, 065602(R) (2006).

[29] M. Conforti, F. Baronio, A. Degasperis, and S. Wabnitz, Parametric frequency conversion of short optical pulses controlled by a CW background Opt. Express 15, 12246 (2007). 
[30] F. Baronio, M. Conforti, A. Degasperis, and S. Wabnitz, Threewave trapponic solitons for tunable highrepetition rate pulse train generation IEEE J. Quantum Electron 44, 542 (2008).

[31] F. Baronio, M. Conforti, M. Andreana, V. Couderc, C. De Angelis, S. Wabnitz, A.Barthelemy, and A. Degasperis, Frequency generation and solitonic decay in three wave interactions Opt. Express 17, 12889 (2009).

[32] F. Baronio, M. Conforti, C. De Angelis, A. Degasperis, M. Andreana, V. Couderc, and A.Barthelemy, Velocity-locked solitary waves in quadratic media Phys. Rev. Lett 104, 113902 (2010).

[33] F. Calogero and A. Degasperis, Spectral Transform and Solitons, Vol. 1, North Holland Publishing, Amsterdam, (1982).

[34] A. Degasperis S. and Lombardo, Exact solutions of the 3-wave resonant interaction equation, Physica D: Nonlinear Phenomena, 214, 157 (2006).

[35] A. Degasperis, S. Lombardo, Multicomponent integrable wave equations I. The Darboux-Dressing Transformations Journal of Physics A: Mathematical and Theoretical, 40, 961 (2007).

[36] A. Degasperis, S. Lombardo, Multicomponent integrable wave equations II. Soliton solutions Journal of Physics A: Mathematical and Theoretical, 42, 385206 (2009).

[37] D.J. Kaup, A. Reiman, and A. Bers, Space-time evolution of nonlinear threewave interactions. I. Interaction in a homogeneous medium Rev. Mod. Phys. 51, 277 (1979). 